() Entomologica Fennica. 19 April 2002

\title{
Ant-associated beetles of Fennoscandia and Denmark
}

\author{
Jussi Päivinen, Petri Ahlroth \& Veijo Kaitala
}

Päivinen, J., Ahlroth, P. \& Kaitala, V. 2002: Ant-associated beetles of Fennoscandia and Denmark. - Entomol. Fennica 13: 20-40.

Ants have a negative impact on populations of many arthropod species. On the other hand, numerous arthropod species live in association with ants. In this paper we list ant-associated beetles (including myrmecophiles) of Fennoscandia and Denmark. Data is based on a literature survey and new field observations. We list 369 ant-associated beetle species of which 73 are categorized as myrmecophilous. Our data suggests that there might be numerous beetle species associated with ants, which are not generally known to do so. This indicates that ant colonies may be important habitats for a large variety of beetle species.

Jussi Päivinen, Department of Biological and Environmental Science, University of Jyväskylä, P.O. Box 35, FIN-40351 Jyväskylä, Finland; E-mail: jupepa@dodo.jyu.fi

Petri Ahlroth, Jyväskylä University Museum, Section of Natural History, P.O. Box 35, FIN-40351 Jyväskylä, Finland; E-mail: pahl@dodo.jyu.fi

Veijo Kaitala, Department of Biological and Environmental Science, University of Jyväskylä, P.O. Box 35, FIN-40351 Jyväskylä, Finland; E-mail: vkaitala@cc.jyu.fi

Received 22 March 2001, accepted 24 October 2001

\section{Introduction}

Several studies suggest that ants, through predation and disturbance, have a negative impact on various arthropods, such as harvestmen (Opiliones), spiders (Araneae) and ground beetles (Carabidae) (Cherix \& Bourne 1980, Rosengren \& Sundström 1991, Niemelä et al. 1992, Laakso \& Setälä 2000). Nevertheless, numerous arthropods live in an association with ants (see the reviews by Larsson 1943, Kistner 1982, Hölldobler \& Wilson 1990). In a recent study in Finland, wood ant mounds (Formicidae, Formica spp.) were considered to be hot spots for earthworms and many arthropods that are not usually considered as ant-associated or myrmecophilous species (Laakso \& Setälä
1997, 1998). According to Laakso \& Setälä (1997), the activity and presence of ants create habitats that have highly different abiotic and biotic conditions compared to the surrounding forest floor. For example, wood ant mounds differ from the surrounding by having a high and regulated temperature, and high input of detritus and arthropod carrion (Rosengren et al. 1987). Moreover, the wood ant mounds are actively shielded from various arthropod and vertebrate predators (e.g. ground beetles, spiders, birds and shrews). Based on these assumptions, we predict that ant colonies may also maintain a high species richness of many other arthropod groups.

Ant-associated insects have evolved different types of symbiotic relationships with ants. "Ant 
guests", commonly known as myrmecophiles, are dependent on ant societies at least during part of their life cycles (Hölldobler \& Wilson 1990). Other species do so occasionally, functioning as casual predators or temporary nest commensals. Both of these ant-associated insect groups include a great variety of springtails (Collembola), beetles (Coleoptera) and butterflies (Lepidoptera), as well as less abundant representatives of a wide range of other insect groups (Hölldobler \& Wilson 1990).

One of the most diverse ant-associated insect taxa are beetles. According to Hölldobler \& Wilson (1990), 35 different ant-associated beetle families, consisting of thousands of species, have hitherto been recorded. Although the literature on ant-associated beetle species is enormous, a large part of the available data consists only of incidental observations or ecological studies of individual species. Only a few detailed lists of the host ants and their myrmecophilous beetles have been previously published (e.g. Johansen 1904, Donisthorpe 1927, Larsson 1943, Collingwood 1957, Wilson 1971, Kistner 1982, Hölldobler \& Wilson 1990, Wojcik 1990, Franck 1992, Kistner et al. 1997), and an updated list on this fauna in Fennoscandia and Denmark is virtually lacking.

In this paper we survey the most comprehensive list of myrmecophilous and other ant-associated beetles in Fennoscandia (Finland, Norway and Sweden) and Denmark. The two groups myrmecophilous and other ant-associated beetles — will be hereafter referred to by the abbreviation AAB (ant-associated beetles). We also demonstrate that ant colonies are species rich habitats for beetles. This knowledge is based on the present literature of beetles and their ecology. The knowledge is reinforced by our own data, and by field observations of several coleopterologists. Finally, we discuss the previous classifications of myrmecophilous beetles.

\section{Material and methods}

In this study, we collected a list of beetle species, which have been observed with ants according to literature and some field observations. We also collected data on host ants and special requirements of beetle species. We used the following sources of information on ant-associated beetles.
Danmarks Fauna: Hansen \& Henriksen (1927), Larsson (1943), Hansen (1950, 1951a, 1951b, 1952, 1954, 1956, 1957, 1958, 1965, 1966a, 1966b, 1968a, 1968b, 1969, 1973a, 1973b, 1973c, 1973d), Die Käfer Mitteleuropas Ökologie: (Koch 1989a, 1989b, 1992) and Svensk Insektfauna: Aurivillius (1917, 1920), Spessivtseff (1925), Lindroth (1933, 1961), Palm (1948, 1961, 1963, 1966, 1968, 1970, 1972) and Landin (1957). In addition to these books, we collected data from some periodicals: Acta Entomologica Fennica, Annales Entomologici Fennici, Entomologica Fennica, Entomologica Scandinavica, Entomologisk Tidskrift, Entomologiske Meddelelser, Entomologist's Gazzette, Norwegian Journal of Entomology, Fauna Norwegica, Notulae Entomologicae. From these periodicals we used the following references. Meinert (1887-88a, 1887-88b, 1889-90), Lovendal (1891-92), Johansen (189596, 1903, 1904, 1906), Schlick (1895-96, 1897), Holstebroe (1910), Adlerz (1911, 1912), Rosenberg (1913, 1914, 1924), West (1913, 1930), Krogerus (1934), Palm (1936, 1943, 1946, 1947, 1954a, 1954b, 1956, 1959, 1979, 1985a, 1985b), Palmen (1936), Kangas (1938, 1951, 1982, 1983), Lindberg (1943), Kryger (1945), Lindgren (1945), Widenfalk, 1954, Kornerup (1960), Collingwood (1957, 1959, 1965), Wegelius (1960), Lundberg (1961, 1972, 1973, 1976, 1977, 1978a, 1978b, 1980, 1981, 1983, 1984, 1993), Hansen (1964, 1967, 1968c, 1970, 1971), Huggert (1967), Skidmore \& Johnson (1969), Huggert \& Ulefors (1971), Baranowski (1975, 1976, 1979, 1980a, 1980b, 1982), Szymzsakowski (1975), Andersson (1977,1981), Nilssen \& Andersen (1977), Rydh (1977), Sörensson (1979, 1996), Bangsholt (1981), Persson (1981), Gillerfors (1982, 1990), Pritzl (1982), Ehnström (1983), Andersen et al. (1984), Hansen \& Mahler (1985), Mahler (1987), Clayhills (1988), Hansen (1988), Johnson (1988), Martin (1989), Hansen et al. (1990, 1991, 1994), Paulsen (1991) and Siitonen (1993). See also Douglas (1858), Adlerz (1913), Donisthorpe (1927), Lindroth (1946), Owen (1986, 2000), Vallenduuk (1987), Franc (1992), Völkl (1995), Sagvolden \& Hansen (1996), Whitehead (1996), Sloggett et al. (1999) and Jorum (2000). Furthermore, our data include field observations from Finland (Päivinen 1999, Mukkala pers. comm., Rutanen pers. comm.). In these field studies, all beetles were captured inside ant mounds and occasional observations were not included in our list.

\section{Results}

Based on the literature survey and field observations a total of 369 ant-associated species of beetles have been recorded in Fennoscandia and Denmark (Table 1). These species numbers include both myrmecophiles and other ant-associated beetles (Table 1). The total number of host ant species was 39. The largest number of beetle species was found 
Table 1. List of the ant associated beetle species and their host ants according to the studied literature and new field observations. ${ }^{*}=$ myrmecophile according to Koch (1989a, 1989b, 1992), and ${ }^{*}=$ myrmecophile according to Szymczakowski (1975). Abbreviations under the column "host ant" are explained in Table 2. Under the column Ref., 1 = Danmarks Fauna (Hansen \& Henriksen 1927, Larsson 1943, Hansen 1950, 1951a, 1951b, 1952, 1954, 1956, 1957, 1958, 1965, 1966a, 1966b, 1968a, 1968b, 1969, 1973a, 1973b, 1973c, 1973d), 2 = Die Käfer Mitteleuropas Ökologie (Koch 1989a, 1989b, 1992), 3 = Svensk Insekt Fauna (Spessivtseff 1925, Landin 1957, Lindroth 1933, 1961, Palm 1948, 1961, 1963, 1966, 1968, 1970, 1972), 4 = Päivinen 1999, 5 = Mukkala pers. comm., 6 = Rutanen pers. comm., 7 = Völkl 1995, 8 = Ehnström 1983, 9 = Szymczakowski 1975, 10 = Adlerz 1911, 11 = Adlerz 1912, 12 = Palm 1936, 13 = Palm 1943, $14=$ Lindgren 1945, 15 = Palm 1946, $16=$ Palm 1947, $17=$ Palm 1954a, $18=$ Palm 1954b, $19=$ Widenfalk 1954, 20 = Palm 1956, 21 = Franc 1992, 22 = Adlerz 1913, 23 = Palm 1959, $24=$ Lundberg 1961, 25 = Huggert 1967, 26 = Huggert \& Ulefors 1971, 27 = Lundberg 1972, 28 = Lundberg 1973, 29 = Baranowski 1975, 30 = Lundberg 1976, 31 = Baranowski 1976, 32 = Andersson 1977, 33 = Rydh 1977, 34 = Lundberg 1978b, 35 = Lundberg 1978a, 36 = Palm 1979, 37 = Sörenssön 1979, 38 = Baranowski 1979, 39 = Owen 1986, 40 = Baranowski 1980a, 41 = Baranowski 1980b, 42 = Persson 1981, $43=$ Lundberg 1981, 44 = Andersson 1981, 45 = Gillerfors 1982, 46 = Baranowski 1982, 47 = Lundberg 1983, 48 = Lundberg 1984, $49=$ Palm 1985a, 50 = Palm 1985b, 51 = Gillerfors 1990, 52 = Lundberg 1993, 53 = Sörensson 1996, 54 = Collingwood 1957, 55 = Collingwood 1959, 56 = Collingwood 1965, 57 = Douglas 1858, 58 = Palmen 1936, 59 = Kangas 1938, 60 = Kangas 1951, 61 = Kangas 1982, 63 = Kangas 1983, 64 = Siitonen 1993, 65 = Krogerus 1934, $66=$ Lindberg 1943, 67 = Wegelius 1960, 68 = Clayhills 1988, 69 = Donisthorpe 1927, 70 = Lundberg 1980, $71=$ Lundberg 1977, $72=$ Skidmore \& Johnson 1969, $73=$ Johnson 1988, $74=$ Whitehead 1996, $75=$ Sloggett et al. 1999, 76 = Owen 2000, 77 = Nilssen \& Andersen 1977, 78 = Andersen et al. 1984, 79 = Paulsen 1991, 80 = Sagvolden \& Hansen 1996, 81 = Johansen 1904, 82 = Meinert 1887-88a, $83=$ Meinert 1889-90, 84 = Lovendal 1891-92, 85 = Johansen 1895-96, 86 = Schlick 1895-96, 87 = Meinert 1887-88b, 88 = Schlick 1897, 89 = Johansen 1903, 90 = Johansen 1906, 91 = Holstebroe 1910, $92=$ West 1913, 93 = Rosenberg 1913, 94 = Rosenberg 1914, 95 = West 1930, 96 = Kryger \& Sonderup 1945, 97 = Kornerup 1960, $98=$ Hansen 1967, 99 = Hansen 1968c, $100=$ Hansen 1970, $101=$ Hansen 1971, 102 = Bangsholt 1981, 103 = Pritzl \& Mahler 1982, 104 = Hansen \& Mahler 1985, 105 = Mahler 1987, 106 = Hansen 1988, $107=$ Martin 1989, 108 = Hansen et al. 1990, $109=$ Hansen et al. 1991, $110=$ Hansen et al. 1994, $111=$ Jorum 2000, 112 = Lindroth 1946, $113=$ Hansen 1964.

\begin{tabular}{|c|c|c|c|}
\hline Ant associated beetle species & Host ant & Special requirement & Ref. \\
\hline \multicolumn{4}{|l|}{ CARABIDAE } \\
\hline Dyschirius globosus (Herbst, 1784) & Lfuli, Frufa & hygrophil & 81 \\
\hline Porotachys bisulcatus (Nicolai, 1822) & Formicidae & & 2 \\
\hline Syntomus truncatellus (Linnaeus, 1761) & Lfuli, Frufa & xerophil & 81 \\
\hline $\begin{array}{l}\text { HYDROPHILIDAE } \\
\text { Megasternum obscurum (Marsham, 1802) }\end{array}$ & Mrubr, Ffusc & hygrophil & 81 \\
\hline \multicolumn{4}{|l|}{ PTILIIDAE } \\
\hline Ptenidium gressneri Erichson, 1845 & $\begin{array}{l}\text { Camponotus spp., } \\
\text { Lfuli, Ffusc }\end{array}$ & hygrophil, mycetophil & $\begin{array}{l}1,2,54,69,81,89 \\
113\end{array}$ \\
\hline Ptenidium laevigatum Erichson, 1845 & Lfuli, Frufa & hygrophil, mycetophil & 1,69 \\
\hline Ptenidium turgidum Thomson, 1855 & Lbrun, Frufa & mycetophil & 1,69 \\
\hline Ptenidium formicetorum* Kraatz, 1851 & $\begin{array}{l}\text { Lbrun, Lfuli, Frufa, } \\
\text { Fpoly, Faqui, Fprat }\end{array}$ & myrmecophil, mycetophil & $\begin{array}{l}1,2,4,5,6,21,49, \\
54,63,69,81,89, \\
112,113\end{array}$ \\
\hline Ptenidium pusillum (Gyllenhal, 1808) & Frufa & mycetophil & 81 \\
\hline Micridium halidaii (Matthews, 1868) & Lbrun, Lnige & mycetophil & 101,113 \\
\hline Ptilium myrmecophilum* (Allibert, 1844) & $\begin{array}{l}\text { Lasius spp., } \\
\text { Fsang, Frufa, } \\
\text { Fprat, Ftrun, } \\
\text { Faqui }\end{array}$ & myrmecophil, mycetophil & $\begin{array}{l}1,2,5,6,21,56 \\
63,69,81,112,113\end{array}$ \\
\hline Ptilium modestum Wankowicz, 1869 & $\begin{array}{l}\text { Lasius spp., Frufa, } \\
\text { Fexse }\end{array}$ & mycetophil & $\begin{array}{l}1,6,27,81,102 \\
113\end{array}$ \\
\hline Pteryx suturalis (Heer, 1841) & Fsuec & mycetophil & 16 \\
\hline
\end{tabular}




\begin{tabular}{|c|c|c|c|}
\hline Ant associated beetle species & Host ant & Special requirement & Ref. \\
\hline Acrotrichis montandonii (Allibert, 1844) & $\begin{array}{l}\text { Lfuli, Lbrun, Lumbr, } \\
\text { Frufa, Fpoly, Fprat, } \\
\text { Faqui }\end{array}$ & mycetophil & $\begin{array}{l}1,2,5,6,56,63 \\
69,113\end{array}$ \\
\hline Acrotrichis dispar (Matthews, 1865) & Frufa & mycetophil & 1 \\
\hline Acrotrichis brevipennis (Erichson, 1845) & Frufa & hygrophil, mycetophil & 81 \\
\hline Acrotrichis pumila (Erichson, 1845) & Frufa & mycetophil & 81 \\
\hline Acrotrichis thoracica (Waltl, 1838) & Frufa & mycetophil & 2 \\
\hline Acrotrichis silvatica Rosskothen, 1935 & Formicidae & mycetophil & 1,113 \\
\hline Acrotrichis norvegica Strand, 1941 & Lbrun & mycetophil & 2 \\
\hline Acrotrichis intermedia (Gillmeister, 1845) & Frufa & mycetophil & 1,113 \\
\hline Acrotrichis atomaria (DeGeer, 1774) & Frufa & mycetophil & $1,81,113$ \\
\hline Acrotrichis fascicularis (Herbst, 1793) & Frufa & mycetophil & $1,81,113$ \\
\hline \multicolumn{4}{|l|}{ AGYRTIDAE } \\
\hline \multicolumn{4}{|l|}{ CHOLEVIDAE } \\
\hline Ptomaphagus sericatus (Chaudoir, 1845) & Formicidae & necrophil & 91 \\
\hline Nemadus colonoides (Kraaz, 1851) & $\begin{array}{l}\text { Lnige, Lbrun, Lfuli, } \\
\text { Frufa }\end{array}$ & & $\begin{array}{l}1,21,81,89,91 \\
93\end{array}$ \\
\hline Eocatops lapponicus ${ }^{* *}$ Szymczakowski, 1975 & Flema & myrmecophil & $9,30,35$ \\
\hline Dreposcia umbrina (Erichson, 1837) & Lbrun, Lfuli & & $1,2,21,91$ \\
\hline \multicolumn{4}{|l|}{ SCYDMAENIDAE } \\
\hline Euthiconus conicicollis (Fair. \& Lab., 1855) & $\begin{array}{l}\text { Lnige, Lbrun, Lfuli, } \\
\text { Frufa }\end{array}$ & myrmecophil & $\begin{array}{l}1,2,21,79,101 \\
102\end{array}$ \\
\hline Eutheia plicata (Gyllenhal, 1813) & $\begin{array}{l}\text { Terra, Lfuli, Fexse, } \\
\text { Frufa, Fprat }\end{array}$ & & $\begin{array}{l}1,2,32,37,54,69, \\
92,95,102,105, \\
111\end{array}$ \\
\hline Eutheia linearis Mulsant, 1861 & Lbrun, Lnige, Frufa & & $1,2,104$ \\
\hline Eutheia scydmaenoides Stephens, 1830 & Lfuli, Frufa & & 1,2 \\
\hline Nevraphes elongatulus (Müller \& Kunze, 1822) & Frufa & & 1 \\
\hline Nevraphes angulatus (Müller \& Kunze, 1822) & Formicidae & hygrophil & 1 \\
\hline Nevraphes talparum Lokay, 1924 & Lnige, Lfuli, Frufa & & $1,17,95$ \\
\hline Nevraphes plicicollis Reitter, 1879 & Lnige, Frufa & & $100,102,105$ \\
\hline Scydmographes helvolus (Schaum, 1844) & Lnige, Lfuli, Frufa & & 1 \\
\hline Scydmoraphes minutus* (Chaudoir, 1845) & $\begin{array}{l}\text { Cherc, Lbrun, Lnige, } \\
\text { Lfuli, Frufa, Fprat }\end{array}$ & myrmecophil & $1,2,18,21,49,58$ \\
\hline Stenichnus scutellaris (Müller \& Kunze, 1822) & Lfuli, Frufa & & 1 \\
\hline Stenichnus pusillus (Müller \& Kunze, 1822) & Lfuli, Frufa & & $1,2,69$ \\
\hline Stenichnus godart' (Latreille, 1806) & $\begin{array}{l}\text { Lbrun, Lfuli, Lnige, } \\
\text { Frufa }\end{array}$ & myrmecophil & $\begin{array}{l}1,2,49,54,69 \\
101,108\end{array}$ \\
\hline Stenichnus collaris (Mueller \& Kunze, 1822) & Lfuli, Frufa & & $1,5,44$ \\
\hline Stenichnus bicolor (Denny, 1825) & $\begin{array}{l}\text { Lbrun, Lnige, Frufa, } \\
\text { Faqui, Fexse }\end{array}$ & & $1,2,6,69,101$ \\
\hline Microscydmus nanus* (Schaum, 1844) & $\begin{array}{l}\text { Lasius spp., Frufa, } \\
\text { Fprat }\end{array}$ & myrmecophil & $1,2,49,58$ \\
\hline Microscydmus minimus* (Chaudoir, 1845) & Lnige, Frufa & myrmecophil & $2,5,6,40$ \\
\hline Euconnus claviger ${ }^{\star}$ Mueller \& Kunze, 1822 & $\begin{array}{l}\text { Lnige, Lfuli, Lbrun, } \\
\text { Frufa, Faqui }\end{array}$ & myrmecophil & $\begin{array}{l}1,2,4,6,21,69 \\
81,95,105,106 \\
111,112\end{array}$ \\
\hline Euconnus pragensis ${ }^{\star}$ (Machulka, 1923) & $\begin{array}{l}\text { Clign, Lnige, } \\
\text { Lbrun, Frufa }\end{array}$ & myrmecophil & $2,21,40,41,80$ \\
\hline
\end{tabular}


Table 1. Continued.

\begin{tabular}{|c|c|c|c|}
\hline Ant associated beetle species & Host ant & Special requirement & Ref. \\
\hline Euconnus maklinit (Mannerheim, 1844) & $\begin{array}{l}\text { Lnige, Lbrun, Lfuli, } \\
\text { Frufa }\end{array}$ & myrmecophil & $\begin{array}{l}2,5,6,21,40,63 \\
95,105,112\end{array}$ \\
\hline Euconnus wetterhallii (Gyllenhal, 1813) & Myrmica spp. & hygrophil & 106 \\
\hline Euconnus denticornis (Mueller \& Kunze, 1822) & Frufa & hygrophil & 1,106 \\
\hline Euconnus hirticollis (Illiger, 1798) & Faqui & hygrophil & 4 \\
\hline Scydmaenus tarsatus Muller \& Kunze, 1822 & Lfuli & & 87 \\
\hline Scydmaenus rufus Müller \& Kunze, 1922 & $\begin{array}{l}\text { Myrmica spp., } \\
\text { Lbrun, Frufa }\end{array}$ & & $1,36,49$ \\
\hline Scydmaenus perrisit (Reitter, 1882) & Lbrun & myrmecophil & $2,19,21,52$ \\
\hline Scydmaenus hellwigit (Herbst, 1792) & $\begin{array}{l}\text { Lbrun, Lfuli, Frufa, } \\
\text { Fpoly }\end{array}$ & myrmecophil & $\begin{array}{l}1,2,5,6,19,21 \\
40,49,63,112\end{array}$ \\
\hline \multicolumn{4}{|l|}{ STAPHYLINIDAE } \\
\hline Gabrius nigritulus (Gravenhorst, 1802) & Formica spp. & hygrophil & 81 \\
\hline Gabrius sphagnicola (Sjöberg, 1950) & Fural & & 30,50 \\
\hline Gabrius splendidulus (Gravenhorst, 1802) & Frufa & & $2,81,113$ \\
\hline Gabrius osseticus (Kolenati, 1846) & Lfuli, Frufa & hygrophil & 81,113 \\
\hline Bisnius subuliformis (Gravenhorst, 1802) & Lfuli & & 1,101 \\
\hline Philonthus ventralis (Gravenhorst, 1802) & Frufa & hygrophil & 1 \\
\hline Platydracus fulvipes (Scopoli, 1763) & Mrugi & hygrophil & 1,113 \\
\hline Platydracus stercorarius (Olivier, 1795) & $\begin{array}{l}\text { Mrubr, Mrugi, } \\
\text { Mscab, Tcaes, } \\
\text { Lalie, Lflav, Frufa, } \\
\text { Prufe }\end{array}$ & xerophil & 1,69 \\
\hline Platydracus latebricola (Gravenhorst, 1806) & $\begin{array}{l}\text { Mrubr, Mrugi, } \\
\text { Lumbr, Lfuli, Frufa }\end{array}$ & xerophil & $1,69,105,113$ \\
\hline Staphylinus erythropterus Linnaeus, 1758 & Myrmica spp. & hygrophil & $1,2,42,113$ \\
\hline Heterothops praevius Erichson, 1840 & Frufa & pholeophil & 81 \\
\hline Heterothops niger Kraaz, 1868 & Lfuli, Frufa & pholeophil & 1,69 \\
\hline Heterothops dissimilis (Gravenhorst, 1802) & Frufa & & $1,56,82,113$ \\
\hline Euryporus picipes (Paykull, 1800) & Lfuli & hygrophil & $1,82,113$ \\
\hline Quedius mesomelinus (Marsham, 1802) & Lfuli, Frufa & phloeophil & 69,81 \\
\hline Quedius maurus (Sahlberg,1830) & Lfuli & & 1,113 \\
\hline Quedius invreai Gridelli, 1924 & Lfuli & pholeophil & 2,41 \\
\hline Quedius ochripennis (Ménétriés, 1832) & Lfuli & & $1,2,113$ \\
\hline Quedius brevicornis Thomson, 1860 & Lasius spp. & & 2 \\
\hline Quedius brevis Erichson, 1840 & $\begin{array}{l}\text { Lfuli, Fsang, Frufa, } \\
\text { Fexse, Faqui, } \\
\text { Flugu }\end{array}$ & myrmecophil & $\begin{array}{l}1,2,4,5,6,21,44 \\
49,54,55,56,57 \\
63,69,81,82,97 \\
111,112,113\end{array}$ \\
\hline Quedius microps (Gravenhorst, 1847) & Cherc, Lnige, Lfuli & & $\begin{array}{l}1,2,40,49,81 \\
112,113\end{array}$ \\
\hline Quedius truncicola Fair. \& Lab., 1855 & Lfuli & & 2,53 \\
\hline Quedius scitus (Gravenhorst, 1806) & Lbrun, Frufa & & $1,2,69,113$ \\
\hline Quedius fuliginosus (Gravenhorst, 1802) & Lfuli & hygrophil & 81 \\
\hline Quedius boops (Gravenhorst, 1802) & Lfuli & hygrophil, xerophil & 81 \\
\hline Leptacinus formicetorum Märkel, 1841 & $\begin{array}{l}\text { Lbrun, Frufa, Fprat, } \\
\text { Fexse, Fpoly, } \\
\text { Faqui, Fural, Frufi }\end{array}$ & myrmecophil & $\begin{array}{l}1,2,3,4,5,6,21 \\
57,63,67,69,81 \\
82,112,113\end{array}$ \\
\hline Gyrohypnus scoticus (Joy, 1913) & Lfuli, Frufa & hygrophil & 81 \\
\hline Gyrohypnus angustatus (Stephens, 1833) & Lfuli, Frufa & hygrophil & $1,49,113$ \\
\hline Gyrohypnus atratus* (Heer, 1839) & $\begin{array}{l}\text { Lfuli, Frufa, Faqui, } \\
\text { Fpoly, Fprat }\end{array}$ & myrmecophil & $\begin{array}{l}1,2,4,5,6,21,54 \\
63,69,81,82,112 \\
113\end{array}$ \\
\hline Nudobius lentus (Gravenhorst, 1806) & Fpoly & & 5 \\
\hline Hypnogyra glabra (Nordmann, 1837) & Lbrun, Lfuli, Frufa & & $1,6,69,113$ \\
\hline
\end{tabular}




\begin{tabular}{|c|c|c|c|}
\hline Ant associated beetle species & Host ant & Special requirement & Ref. \\
\hline Xantholinus linearis (Olivier, 1795) & Lfuli, Frufa & hygrophil & 1,113 \\
\hline Xantholinus meyeriDrugmand & Lfuli, Frufa & hygrophil & 1 \\
\hline Xantholinus tricolor (Fabricius, 1787) & Lfuli, Fsang, Frufa & & 81,113 \\
\hline Xantholinus laevigatus Jacobson, 1847 & Lfuli, Frufa & hygrophil & $1,81,113$ \\
\hline Othius punctulatus (Goeze, 1777) & Formicidae & & 81 \\
\hline Othius angustus Stephens, 1833 & Lfuli & xerophil & 81 \\
\hline Othius myrmecophilus Kiesenwetter, 1843 & $\begin{array}{l}\text { Mscab, Lfuli, Lbrun, } \\
\text { Fexse, Fsang, Frufa }\end{array}$ & & $1,54,69,97$ \\
\hline Astenus gracilis (Paykull, 1789) & Formica spp. & xerophil & 2 \\
\hline Medon apicalis (Kraatz, 1857) & Frufa & pholeophil & $1,2,113$ \\
\hline Medon rufiventris (Nordmann, 1837) & Lfuli & xerophil & 71 \\
\hline Medon castaneus (Gravenhorst, 1802) & Formicidae & phloeophil & 81 \\
\hline Medon fusculus (Mannerheim, 1830) & Formicidae & pholeophil & 2 \\
\hline Sunius melanocephalus (Fabricius, 1792) & Lfuli & xerophil & 2,81 \\
\hline Sunius bicolor (Olivier, 1795) & Mrugi, Lflav & hygrophil, xerophil & 1,69 \\
\hline Scopaeus laevigatus (Gyllenhal, 1827) & Frufa & hygrophil & 1 \\
\hline Scopaeus minutus Erichson, 1840 & Fural & thermophil, xerophil & 1 \\
\hline Scopaeus pusillus Kiesenwetter, 1843 & Frufa & thermophil, xerophil & 2 \\
\hline Stenus aterrimus ${ }^{\star}$ Erichson, 1839 & Frufa, Fprat, & myrmecophil & $\begin{array}{l}1,2,21,25,37,70 \\
81,102,112,113\end{array}$ \\
\hline Stenus crassus Stephens, 1833 & Frufa & hygrophil & 1 \\
\hline Hapalaraea nigra (Gravenhorst, 1806) & Lfuli & & 1,113 \\
\hline Omalium caesum Gravenhorst, 1806 & Lfuli, Frufa & & 81 \\
\hline Xylodromus depressus (Gravenhorst, 1802) & Formicidae & & 81 \\
\hline Xylodromus affinis (Gerhardt, 1877) & Lfuli & & 1,113 \\
\hline Anotylus rugosus (Fabricius, 1775) & Formica spp. & hygrophil & 1 \\
\hline Platystethus arenarius (Fourcroy, 1785) & Frufa & coprophil & 81 \\
\hline Bledius procerulus Erichson, 1840 & Lflav & xerophil, psammophil & 2 \\
\hline Trichophya pilicornis Gyllenhal, 1810) & Faqui & pholeophil & 4 \\
\hline Mycetoporus lepidus (Gravenhorst, 1806) & Lfuli, Frufa & & 2,81 \\
\hline Ischnosoma bergrothi (Hellen, 1925) & Myrmica spp. & tyrphophil & 3 \\
\hline Carphacis striatus (Olivier, 1794) & Formicidae & mycetophil & 1 \\
\hline Bolitobius cingulatus Mannerheim, 1830 & Myrmica spp. & hygrophil & 3 \\
\hline Sepedophilus testaceus (Fabricius, 1792) & Lfuli, Frufa & pholeophil, mycetophil & 2,81 \\
\hline Sepedophilus marshami(Stephens, 1832) & Lasius spp., Faqui & mycetophil & 4,6 \\
\hline Sepedophilus immaculatus (Stephens, 1832) & Lfuli, Frufa & & 81 \\
\hline Tachyporus nitidulus (Fabricius, 1781) & Fpoly & & 5 \\
\hline Tachyporus obtusus (Linnaeus, 1767) & Frufa & & 81 \\
\hline Tachyporus hypnorum (Fabricius, 1775) & Fpoly & & 5 \\
\hline Tachyporus chrysomelinus (Linnaeus, 1758) & Fpoly & & 5 \\
\hline Tachyporus scitulus (Erichson, 1839) & Fexse & xerophil & 2 \\
\hline Tachyporus corpulentus J. Sahlberg, 1876 & Frufa & xerophil & 1 \\
\hline Tachinus rufipes (Linnaeus, 1758) & Lfuli, Frufa & saprophil & 81 \\
\hline Tachinus fimetarius Gravenhorst, 1802 & Lfuli, Frufa & & 81 \\
\hline Tachinus marginellus (Fabricius, 1781) & Lfuli, Frufa & saprophil & 81 \\
\hline Lamprinodes saginatus* (Gravenhorst, 1806) & $\begin{array}{l}\text { Mrugi, Msabu, } \\
\text { Mscab, Mrubr, } \\
\text { Lflav, Lfuli, Ffusc, } \\
\text { Frufa, Fexse, } \\
\text { Fsang }\end{array}$ & xerophil, myrmecophil & $\begin{array}{l}1,2,21,26,38,69 \\
81,113\end{array}$ \\
\hline Aleochara spissicornis Erichson, 1839 & Tcaes & thermophil & 2 \\
\hline Aleochara moesta Gravenhorst, 1802 & F. rufa & & 85 \\
\hline
\end{tabular}


Table 1. Continued.

\begin{tabular}{|c|c|c|c|}
\hline Ant associated beetle species & Host ant & Special requirement & Ref. \\
\hline Aleochara lanuginosa Gravenhorst, 1802 & Frufa & coprophil & 81,85 \\
\hline Aleochara lygaea Kraatz, 1862 & Lfuli & coprophil & $1,95,113$ \\
\hline Aleochara villosa Mannerheim, 1830 & Formicidae & coprophil & 1 \\
\hline Aleochara sanguinea (Linnaeus, 1758) & Lbrun, Lfuli & coprophil & $1,69,110$ \\
\hline Aleochara spadicea (Erichson, 1839) & Lfuli & & 3 \\
\hline Aleochara ruficornis Gravenhorst, 1802 & Lfuli, Frufa, Ffusc & & $1,69,81$ \\
\hline Oxypoda opaca (Gravenhorst, 1802) & Lfuli & & $1,81,113$ \\
\hline Oxypoda longipes Mulsant \& Rey, 1861 & Lfuli & & 1,113 \\
\hline Oxypoda vittata* Märkel, 1842 & Lfuli, Frufa & myrmecophil & $\begin{array}{l}1,2,13,21,32,44, \\
49,69,81,85,95, \\
101,112,113\end{array}$ \\
\hline Oxypoda acuminata (Stephens, 1832) & Lfuli, Lnige & hygrophil & 1,81 \\
\hline Oxypoda spectabilis Märkel, 1844 & Lfuli & hygrophil & 1 \\
\hline Oxypoda umbrata (Gyllenhal, 1810) & Lfuli & hygrophil & 81 \\
\hline Oxypoda hansseniStrand, 1946 & Flema & & 68,70 \\
\hline Oxypoda abdominalis (Mannerheim, 1830) & Formicidae & xerophil & 2 \\
\hline Oxypoda togata Erichson, 1837 & Lasius spp. & psammophil & 2 \\
\hline Oxypoda exoleta Erichson, 1839 & Lfuli & xerophil & 105,106 \\
\hline Oxypoda recondita Kraatz, 1856 & $\begin{array}{l}\text { Myrmicaspp., } \\
\text { Lfuli, Lbrun, Frufa, } \\
\text { Fsang }\end{array}$ & & $1,2,3,69,81,113$ \\
\hline Oxypoda serpentata Kangas, 1983 & Frufa coll. & & 63 \\
\hline Oxypoda arborea Zerche, 1994 & Lfuli & & 1,113 \\
\hline Oxypoda testacea Erichson, 1839 & Frufa & & 2 \\
\hline Oxypoda brachyptera (Stephens, 1832) & Frufa, Tcaes & xerophil & 2 \\
\hline Oxypoda annularis (Mannerheim, 1830) & Frufa & & $1,2,67,81,85,113$ \\
\hline Oxypoda flavicornis Kraatz, 1856 & Faqui & hygrophil & 4 \\
\hline Oxypoda advena Mäklin, 1846 & Lfuli & & 1 \\
\hline Oxypoda ferruginea Erichson, 1839 & F. rufa & hygrophil & 85 \\
\hline Oxypoda sororThomson, 1855 & Lfuli & hygrophil & 1,113 \\
\hline Oxypoda formiceticola* Märkel, 1841 & $\begin{array}{l}\text { Lasius spp., Ffusc, } \\
\text { Fpoly, Fexse, Frufa, } \\
\text { Faqui, Flugu }\end{array}$ & myrmecophil & $\begin{array}{l}1,2,3,4,5,6,21 \\
39,56,63,69,81 \\
85,111,112,113\end{array}$ \\
\hline Oxypoda pratensicola* Lohse, 1967 & $\begin{array}{l}\text { Fexse, Fprat, } \\
\text { Fnigr }\end{array}$ & xerophil, myrmecophil & $2,21,70$ \\
\hline Oxypoda rugicollis* Kraatz, 1856 & $\begin{array}{l}\text { Lasiusspp., Fexse, } \\
\text { Frufa, Fprat }\end{array}$ & myrmecophil & $2,3,5,6,21,24$ \\
\hline Oxypoda haemorrhoa* Mannerheim, 1830 & $\begin{array}{l}\text { Lfuli, Fpoly, } \\
\text { Faqui, Fexse, Fsuec, } \\
\text { Fsang, Frufa, Fprat, } \\
\text { Ftrun, Flugu, Fnigr }\end{array}$ & myrmecophil & $\begin{array}{l}2,3,4,5,6,16,39 \\
56,57,63,67,69 \\
70,81,85,112\end{array}$ \\
\hline Parocyusa rubicunda (Erichson, 1837) & Lnige & hygrophil & $1,81,113$ \\
\hline Stichoglossa semirufa (Erichson, 1839) & Lfuli & & $1,2,113$ \\
\hline Ischnoglossa prolixa (Gravenhorst, 1802) & Lfuli & & 2 \\
\hline Thiasophila angulata* (Erichson, 1837) & $\begin{array}{l}\text { Lfuli, Lbrun, Frufa, } \\
\text { Fprat, Faqui, Fpoly, } \\
\text { Fsang, Fural, Flugu }\end{array}$ & myrmecophil & $\begin{array}{l}1,2,3,4,5,6,21 \\
39,56,57,63,67 \\
69,81,85,112,113\end{array}$ \\
\hline Thiasophila canaliculata* Mulsant \& Rey, 1874 & Frufa, Fexse & myrmecophil & $\begin{array}{l}1,2,33,63,81,85 \\
112,113\end{array}$ \\
\hline Thiasophila wockit (Schneider, 1862) & Cvagu, Cherc & myrmecophil & $\begin{array}{l}2,3,15,18,21,40 \\
98,105,112\end{array}$ \\
\hline
\end{tabular}




\begin{tabular}{|c|c|c|c|}
\hline Ant associated beetle species & Host ant & Special requirement & Ref. \\
\hline Thiasophila bercionis* Bernhauer, 1926 & Fural, Fexse & myrmecophil & $2,23,27,65$ \\
\hline Thiasophila inquilina* Märkel, 1844 & Lfuli, Frufa, Fprat & myrmecophil & $\begin{array}{l}1,2,3,21,43,44 \\
49,69,81,85,95 \\
101,102,105,112 \\
113\end{array}$ \\
\hline Thiasophila lohset Zerche, 1987 & Fprat & myrmecophil & 2,21 \\
\hline Crataraea suturalis (Mannerheim, 1830) & Lfuli, Frufa & & 1,113 \\
\hline Haploglossa gentilis (Märkel, 1844) & Lbrun, Lfuli & & $1,2,21,49,69,113$ \\
\hline Haploglossa villosula (Stephens, 1832) & $\begin{array}{l}\text { Myrmica spp., } \\
\text { Lbrun, Lfuli, Frufa }\end{array}$ & & $\begin{array}{l}1,2,3,21,49,69 \\
81,85,105,113\end{array}$ \\
\hline Haploglossa picipennis (Gyllenhal, 1827) & Lbrun & & 1,113 \\
\hline Haploglossa marginalis (Gravenhorst, 1806) & Lbrun. Lfuli & & $1,21,105,113$ \\
\hline Poromniusa procidua (Erichson, 1837) & Frufa & & 1,113 \\
\hline Ocalaea badia Erichson 1837 & Lfuli, Frufa & hygrophil & 81,85 \\
\hline Ilyobates subopacus Palm, 1935 & Myrmica spp. & hygrophil & 1,3 \\
\hline Ilyobates nigricollis (Paykull, 1800) & Myrmicaspp., L.fuli & hygrophil & 1,81 \\
\hline Amarochara umbrosa (Erichson, 1837) & Lasius spp. & & 1,113 \\
\hline Amarochara bonnairet (Fauvel, 1865) & Lbrun, Lfuli & myrmecophil & $1,2,21,113$ \\
\hline Amarochara forticornis (Lacordaire, 1835) & Lfuli & thermophil & 105 \\
\hline Dinarda dentata* (Gravenhorst, 1806) & $\begin{array}{l}\text { Ffusc, Fsang, Frufi, } \\
\text { Fexse, Fcine, Faqui }\end{array}$ & myrmecophil & $\begin{array}{l}1,2,4,21,69,81 \\
85,105,112,113\end{array}$ \\
\hline Dinarda hagensit Wasmann, 1889 & Fexse, Fprat & myrmecophil & $\begin{array}{l}1,2,21,37,69 \\
105,112,113\end{array}$ \\
\hline Dinarda maerkelit Kiesenwetter, 1843 & $\begin{array}{l}\text { Frufa, Fprat, Fpoly, } \\
\text { Ftrun, Flugu, Fsang }\end{array}$ & myrmecophil & $\begin{array}{l}1,2,3,5,21,37 \\
54,56,69,81,112, \\
113\end{array}$ \\
\hline Meotica exilis (Knoch, 1806) & Formica spp. & hygrophil, pholeophil & 2,85 \\
\hline Ousipalia caesula (Erichson, 1839) & Lfuli, Frufa & psammophil & 81 \\
\hline Aloconota sulcifrons (Stephens, 1832) & Formicidae & hygrophil & 81 \\
\hline Liogluta micans (Mulsant \& Rey, 1852) & Lasius spp. & hygrophil & 6 \\
\hline Liogluta longiuscula (Gravenhorst, 1802) & Lbrun. Lfuli, Frufa & hygrophil & 1 \\
\hline Liogluta alpestris (Heer, 1839) & Lbrun, Lfuli, Ffusc & hygrophil & $1,69,81,113$ \\
\hline Geostiba circellaris (Gravenhorst, 1806) & Lfuli, Frufa & hygrophil & $1,2,85,93$ \\
\hline Callicerus obscurus Gravenhorst, 1802 & Lfuli & hygrophil, pholeophil & 3 \\
\hline Callicerus rigidicornis Erichson, 1839 & Lfuli, Lnige, Frufa & & 1,69 \\
\hline Atheta talpa* (Heer, 1841) & $\begin{array}{l}\text { Lfuli, Frufa, Fprat, } \\
\text { Fpoly, Faqui, Ftrun, } \\
\text { Fexse, Flugu }\end{array}$ & myrmecophil & $\begin{array}{l}1,2,3,4,6,21,39, \\
54,56,63,81,85, \\
111,112,113\end{array}$ \\
\hline Atheta nigra (Kraatz, 1856) & Frufa & & 81 \\
\hline Atheta myrmecobia (Kraatz, 1856) & Lasius spp., Frufa & hygrophil & $\begin{array}{l}1,2,6,81,85,90 \\
95,113\end{array}$ \\
\hline Atheta fungi (Gravenhorst, 1806) & Formicidae & & $81,85,113$ \\
\hline Atheta scapularis (Sahlberg, 1831) & Mrubr, Frufa & mycetophil & $3,85,113$ \\
\hline Atheta sodalis (Erichson, 1837) & $\begin{array}{l}\text { Lfuli, Lbrun, Fexse, } \\
\text { Frufa }\end{array}$ & mycetophil & $\begin{array}{l}1,44,69,81,85 \\
113\end{array}$ \\
\hline Atheta trinotata (Kraatz, 1856) & Lfuli, Frufa & & 81 \\
\hline Atheta flavipes* (Gravenhorst, 1806) & $\begin{array}{l}\text { Lflav, Fexse, Fsang, } \\
\text { Frufa, Fprat, Fpoly, } \\
\text { Faqui, Ftrun, Flugu }\end{array}$ & myrmecophil & $\begin{array}{l}1,2,3,4,5,6,21 \\
42,56,63,69,81 \\
85,93,112,113\end{array}$ \\
\hline
\end{tabular}


Table 1. Continued.

\begin{tabular}{|c|c|c|c|}
\hline Ant associated beetle species & Host ant & Special requirement & Ref. \\
\hline Atheta confusa* (Märkel, 1844) & Lfuli, Frufa & myrmecophil & $\begin{array}{l}1,2,3,21,41,44 \\
49,69,81,85,95 \\
101,102,113\end{array}$ \\
\hline Atheta longicornis (Gravenhorst, 1802) & Formicidae & & 81 \\
\hline Atheta subterranea (Mulsant \& Rey, 1853) & Formicidae & pholeophil & 1,113 \\
\hline Atheta brunneipennis (Thomson, 1852) & Fexse & & 3 \\
\hline Atheta castanoptera (Mannerheim, 1830) & Lfuli, Frufa & mycetophil & 81 \\
\hline Atheta fungicola (Thomson, 1852) & Lfuli, Frufa & mycetophil & 81 \\
\hline Atheta brunnea (Fabricius, 1798) & Lfuli & & 1,69 \\
\hline Atheta hepatica (Erichson, 1839) & Lfuli & & $1,90,113$ \\
\hline Lyprocorrhe anceps ${ }^{*}$ (Erichson, 1837) & $\begin{array}{l}\text { Lfuli, Fexse, Frufa, } \\
\text { Fprat, Faqui, Ftrun, } \\
\text { Flugu, Fnigr }\end{array}$ & myrmecophil & $\begin{array}{l}1,2,4,5,6,21,39, \\
42,56,69,70,81, \\
85,112,113\end{array}$ \\
\hline Acrotona consanguinea (Eppelsheim, 1875) & Lfuli, Lumbr & & 1 \\
\hline Acrotona aterrima (Gravenhorst, 1802) & Formicidae & & 81 \\
\hline Acrotona parvula (Mannerheim, 1830) & Lfuli, Frufa & & 81 \\
\hline Coprothassa melanaria (Mannerheim, 1830) & Formicidae & & 81 \\
\hline Amischa nigrofusca (Stephens, 1832) & Lfuli & & 81 \\
\hline Amischa analis (Gravenhorst, 1802) & $\begin{array}{l}\text { Mscab, Mrubr, Lfuli, } \\
\text { Lbrun, Lflav, Fexse, } \\
\text { Frufa }\end{array}$ & & $1,54,56,81,85$ \\
\hline Amischa bifoveolata (Mannerheim, 1830) & Lfuli, Frufa & & 81 \\
\hline Thamiaraea hospita (Märkel, 1844) & Lfuli & & 81 \\
\hline Drusilla canaliculata* (Fabricius, 1787) & $\begin{array}{l}\text { Mscab, Mrugi, } \\
\text { Mrubr, Msulc, } \\
\text { Tcaes, Lacer, } \\
\text { Lnige, Lfuli, Lflav, } \\
\text { Lbrun, Lalie, Fsang, } \\
\text { Ffusc, Fexse, Frufa }\end{array}$ & xerophil, myrmecophil & $\begin{array}{l}1,2,5,56,69,81 \\
85,97,113\end{array}$ \\
\hline Zyras collaris (Paykull, 1800) & $\begin{array}{l}\text { Mrubr, Mrugi, } \\
\text { Lfuli, Frufa }\end{array}$ & hygrophil & $\begin{array}{l}1,2,3,69,81,85 \\
97,101,112,113\end{array}$ \\
\hline Zyras limbatus* (Paykull, 1789) & $\begin{array}{l}\text { Mrubr, Mscab, } \\
\text { Lfuli, Lflav, Lbrun, } \\
\text { Lnige, Ffusc, } \\
\text { Fsang, Fexse }\end{array}$ & xerophil, myrmecophil & $\begin{array}{l}1,2,3,5,21,54, \\
56,69,81,85,101 \\
112,113\end{array}$ \\
\hline Zyras funestus (Gravenhorst, 1806) & Lfuli & myrmecophil & $\begin{array}{l}1,2,3,12,21,44 \\
49,56,69,81,85 \\
101,112,113\end{array}$ \\
\hline Zyras humeralis* (Gravenhorst, 1802) & $\begin{array}{l}\text { Lbrun, Lumbr, } \\
\text { Lfuli, Frufa, Fprat, } \\
\text { Faqui }\end{array}$ & myrmecophil & $\begin{array}{l}1,2,3,4,5,21,44, \\
49,54,63,69,81, \\
85,97,101,112, \\
113\end{array}$ \\
\hline Zyras cognatus* (Märkel, 1842) & $\begin{array}{l}\text { Lbrun, Lnige, Lfuli, } \\
\text { Ffusc, Fexse }\end{array}$ & myrmecophil & $\begin{array}{l}1,2,3,12,21,44 \\
49,69,81,85,88 \\
101,112,113\end{array}$ \\
\hline Zyras lugens* (Gravenhorst, 1802) & Lbrun, Lfuli & myrmecophil & $\begin{array}{l}1,2,3,6,12,21 \\
44,49,69,81,85 \\
101,112,113\end{array}$ \\
\hline Zyras laticollis* (Märkel, 1844) & Lfuli & myrmecophil & $\begin{array}{l}1,2,3,6,12,21 \\
44,49,69,81,85 \\
101,112,113\end{array}$ \\
\hline Lomechusoides strumosus* (Fabricius, 1792) & Fsang, Frufa, Fprat & xerophil, myrmecophil & $\begin{array}{l}1,2,3,21,69,78, \\
81,85,90,95,105 \\
109,112,113\end{array}$ \\
\hline
\end{tabular}




\begin{tabular}{|c|c|c|c|}
\hline Ant associated beetle species & Host ant & Special requirement & Ref. \\
\hline Lomechusoides wellenii (Palm, 1949) & Fural, Flugu, Frufa & & $3,28,30$ \\
\hline Lomechusoides inflatus (Zetterstedt, 1828) & $\begin{array}{l}\text { Fgaga, Fexse, } \\
\text { Fural, Frufa, Fprat }\end{array}$ & & $3,24,30,48,66$ \\
\hline Lomechusa emarginata* (Paykull, 1789) & $\begin{array}{l}\text { Mrubr, Mrugi, } \\
\text { Msabu, Msulc, } \\
\text { Mrugu, Mscab, } \\
\text { Lasius spp., Ffusc, } \\
\text { Frufa, Fsang }\end{array}$ & myrmecophil & $\begin{array}{l}1,2,3,6,21,22 \\
38,54,56,81,69 \\
72,85,97,112,113\end{array}$ \\
\hline Lomechusa paradoxa* Gravenhorst, 1806 & $\begin{array}{l}\text { Mrubr, Mrugi, } \\
\text { Mscab, Mrugu, } \\
\text { Ffusc, Frufi, Fcuni, } \\
\text { Swest }\end{array}$ & xerophil, myrmecophil & $\begin{array}{l}1,2,21,22,56,69 \\
81,85,105,112 \\
113\end{array}$ \\
\hline Lomechusa pubicollis Brisout de B., 1860 & $\begin{array}{l}\text { Mrubr, Mrugi, } \\
\text { Msulc, Tcaes, Lfuli, } \\
\text { Lnige, Lalie, Lflav, } \\
\text { Lumbr, Ftrun, Frufi, } \\
\text { Ffusc, Frufa }\end{array}$ & myrmecophil & $\begin{array}{l}1,2,3,6,21,22 \\
42,64,112,113\end{array}$ \\
\hline Leptusa ruficollis (Erichson, 1839) & Formica spp. & & 1,2 \\
\hline Tachyusida gracilis* (Erichson, 1837) & Lbrun, Lnige & & $2,69,113$ \\
\hline Euryusa castanoptera Kraatz, 1856 & Lbrun, Lfuli & & $1,2,113$ \\
\hline Euryusa optabilis* Heer, 1839 & $\begin{array}{l}\text { Lbrun, Lnige, Lfuli, } \\
\text { Frufa }\end{array}$ & myrmecophil & $\begin{array}{l}1,2,3,21,44,52 \\
69,76,113\end{array}$ \\
\hline Euryusa sinuata* Erichson, 1837 & Lbrun, Lfuli & myrmecophil & $\begin{array}{l}1,2,17,21,45,52 \\
69,74,113\end{array}$ \\
\hline Euryusa coarctata* Märkel, 1844 & Lbrun & myrmecophil & $1,2,52,113$ \\
\hline Oligota muensteriBernhauer, 1923 & Lasius spp., Frufa & & $3,6,26,29,59$ \\
\hline Oligota tantilla Mennerheim, 1843 & Lasius spp. & & 6 \\
\hline Oligota pusillima (Gravenhorst, 1806) & Lfuli, Frufa, Fexse & & $\begin{array}{l}1,2,3,6,27,59 \\
67,69,81,102,103\end{array}$ \\
\hline Oligota uralensicola Kangas, 1982 & Fural & & 61 \\
\hline Cypha nitida (Palm, 1935) & Lbrun & & 113 \\
\hline Cypha hanseni (Palm, 1949) & Lfuli & & Reference missing \\
\hline Cypha pulicaria (Erichson, 1839) & Formicidae & & Reference missing \\
\hline \multicolumn{4}{|l|}{ PSELAPHIDAE } \\
\hline Meliceria tragardhiPalm, 1938 & Lbrun & & 34 \\
\hline Euplectus nanus (Reichhenbach, 1816) & Lnige, Frufa, Fpoly & hygrophil & $2,5,63,101$ \\
\hline Euplectus kirbiïDanny, 1825 & Lfuli, Lnige & hygrophil & $81,82,101$ \\
\hline Euplectus piceus Motschulsky, 1835 & Lbrun, Frufa & hygrophil & $1,2,54,113$ \\
\hline Euplectus decipiens Raffray, 1910 & Lasius spp. & hygrophil & 6 \\
\hline Euplectus infirmus Raffray, 1910 & Lbrun, Lnige & hygrophil & $99,101,105$ \\
\hline Euplectus sanquineus Motschulsky, 1835 & $\begin{array}{l}\text { Lbrun, Lnige, } \\
\text { Ffusc, Fsang }\end{array}$ & hygrophil & 1 \\
\hline Euplectus signatus (Reichenbach, 1816) & $\begin{array}{l}\text { Lasius spp., Frufa, } \\
\text { Faqui }\end{array}$ & hygrophil & $2,4,6,39,60$ \\
\hline Euplectus bonvouloirissp. rosae Raffray, 1910 & Lfuli & hygrophil & 31 \\
\hline Euplectus punctatus Mulsant, 1861 & Frufa & hygrophil & 5,60 \\
\hline Euplectus karstenii (Reichhenbach, 1816) & Lfuli, Frufa & hygrophil & $1,2,5,6,81$ \\
\hline Euplectus fauveli Guillebeau, 1888 & Frufa & hygrophil & 5,60 \\
\hline Euplectus brunneus (Grimmer, 1841) & $\begin{array}{l}\text { Formica spp., } \\
\text { Lbrun }\end{array}$ & hygrophil & $1,2,113$ \\
\hline Plectophloeus nitidus (Fairmaire, 1857) & Lbrun, Lfuli & hygrophil & $1,2,113$ \\
\hline Saulcyella schmidtii (Märkel, 1844) & Lbrun, Lfuli, Frufa & hygrophil & 2,21 \\
\hline
\end{tabular}


Table 1. Continued.

\begin{tabular}{|c|c|c|c|}
\hline Ant associated beetle species & Host ant & Special requirement & Ref. \\
\hline Trimium brevicorne (Reichenbach, 1816) & $\begin{array}{l}\text { Lasius spp., } \\
\text { Fsuec, Frufa }\end{array}$ & hygrophil & $1,2,16,81$ \\
\hline Batrisodes delaportt' (Aubé, 1833) & Lbrun, Lfuli & myrmecophil & $\begin{array}{l}1,2,19,21,52,69, \\
113\end{array}$ \\
\hline Batrisodes venustus* (Reichhenbach, 1816) & $\begin{array}{l}\text { Mscab, Clign, } \\
\text { Lbrun, Lfuli, Lnige, } \\
\text { Frufa, Ffusc }\end{array}$ & myrmecophil & $\begin{array}{l}1,2,19,21,54,69, \\
81,96,113\end{array}$ \\
\hline Batrisodes hubenthalt Reitter, 1913 & Lbrun, Lnige & myrmecophil & $2,21,29$ \\
\hline Batrisodes adnexus ${ }^{\star}$ (Hampe, 1863) & $\begin{array}{l}\text { Myrmica spp., } \\
\text { Camponotus spp., } \\
\text { Lbrun }\end{array}$ & myrmecophil & $\begin{array}{l}1,2,21,51,69,81, \\
105,113\end{array}$ \\
\hline Bryaxis curtisii (Leach, 1817) & Lfuli & hygrophil & 1,113 \\
\hline Trichonyx sulcicollis (Reichenbach, 1816) & $\begin{array}{l}\text { Myrmica spp., } \\
\text { Lbrun, Lfuli }\end{array}$ & hygrophil & $\begin{array}{l}1,2,21,69,81,90, \\
113\end{array}$ \\
\hline Amauronyx maerkeli (Aubé, 1844) & $\begin{array}{l}\text { Myrmicaspp., } \\
\text { Lbrun, Lflav, Lfuli, } \\
\text { Ffusc, Tcaes }\end{array}$ & hygrophil & $\begin{array}{l}1,2,21,54,69,81, \\
113\end{array}$ \\
\hline Tychus niger (Paykull, 1800) & Lfuli & hygrophil & $1,81,113$ \\
\hline Brachyg/uta fossulata (Reichhenbach, 1816) & Frufa & hygrophil & 5 \\
\hline Chennium bituberculatum* Latreille, 1807 & Tcaes & xerophil, myrmecophil & $2,21,47$ \\
\hline Tyrus mucronatus (Panzer, 1803) & $\begin{array}{l}\text { Lbrun, Lnige, } \\
\text { Ffusc, Frufa, Fsang }\end{array}$ & hygrophil & $2,102,113$ \\
\hline Claviger testaceus ${ }^{\star}$ Preyssler, 1790 & $\begin{array}{l}\text { Mrubr, Mscab, } \\
\text { Tcaes, Lbrun, } \\
\text { Lumbr, Lmixt, Lalie, } \\
\text { Lfuli, Lnige, Lflav }\end{array}$ & xerophil, myrmecophil & $\begin{array}{l}1,2,6,12,21,22 \\
54,69,72,81,105 \\
112,113\end{array}$ \\
\hline Claviger longicornis ${ }^{*}$ Müller, 1818 & $\begin{array}{l}\text { Lumbr, Lnige, Lfuli, } \\
\text { Lbrun, Lflav, Lmixt }\end{array}$ & myrmecophil & $\begin{array}{l}1,2,14,21,46,69 \\
81,94,95,105, \\
112,113\end{array}$ \\
\hline \multicolumn{4}{|l|}{ HISTERIDAE } \\
\hline Abraeus granulum Erichson, 1839 & Lasius spp. & & 2 \\
\hline Abraeus perpusillus (Marsham,1802) & Lfuli, Lbrun, Frufa & & $1,2,81,113$ \\
\hline Abraeus parvulus* Aubé, 1842 & Lasius spp. & myrmecophil & 2 \\
\hline Plegaderus caesus (Herbst, 1792) & Lfuli & & 1,113 \\
\hline Plegaderus dissectus Erichson, 1839 & Formicidae & & 2 \\
\hline Acritus minutus (Herbst, 1792) & Lasius spp. & & 2 \\
\hline Acritus homoeopathicus Wollaston, 1857 & Fprat & & 2 \\
\hline Aeletes atomarius (Aubé, 1842) & Lnige, Formicaspp. & myrmecophil & 2,100 \\
\hline Gnathonchus rotundatus (Kugelann, 1792) & Lfuli & & 1,81 \\
\hline Myrmetes paykullt Kanaar, 1979 & $\begin{array}{l}\text { Lasius spp., Frufa, } \\
\text { Fprat, Fpoly, Faqui }\end{array}$ & myrmecophil & $\begin{array}{l}1,2,4,6,21,54, \\
69,77,95,112,113\end{array}$ \\
\hline Dendrophilus corticalis (Paykull, 1798) & $\begin{array}{l}\text { Lfuli, Lbrun, } \\
\text { Fexec, Frufa }\end{array}$ & & $1,2,69,81,113$ \\
\hline Dendrophilus pygmaeus* (Linnaeus, 1758) & $\begin{array}{l}\text { Lfuli, Fpoly, Frufa, } \\
\text { Fprat, Fexse, } \\
\text { Fpoly, Faqui }\end{array}$ & myrmecophil & $\begin{array}{l}1,2,4,5,6,21,54, \\
56,57,69,78,81 \\
111,112,113\end{array}$ \\
\hline Paromalus flavicornis (Herbst, 1792) & Lbrun, Lfuli & & 1,113 \\
\hline Margarinotus merdarius (Hoffmann, 1803) & Lnige, Lfuli & & $1,12,81$ \\
\hline Hister unicolor Linnaeus, 1758 & Lfuli & saprophil & 1,81 \\
\hline Hister helluo Truqui, 1852 & Formicidae & & 2 \\
\hline Atholus corvinus (Germar, 1817) & Formicidae & xerophil & 2 \\
\hline
\end{tabular}




\begin{tabular}{|c|c|c|c|}
\hline Ant associated beetle species & Host ant & Special requirement & Ref. \\
\hline Hetaerius ferrugineus ${ }^{\star}$ (Olivier, 1789) & $\begin{array}{l}\text { Mscab, Lacer, } \\
\text { Terra, Lfuli, Lnige, } \\
\text { Lflav, Ffusc, Fprat, } \\
\text { Frufi, Fsang, Frufa, } \\
\text { Fcine, Fexse }\end{array}$ & myrmecophil, xerophil & $\begin{array}{l}1,2,21,37,38,69 \\
81,105,113\end{array}$ \\
\hline \multicolumn{4}{|l|}{ CLAMBIDAE } \\
\hline Clambus minutus & Frufa & hygrophil, mycetophil & 1 \\
\hline \multicolumn{4}{|l|}{ SCARABAEIDAE } \\
\hline Diastictus vulneratus (Sturm, 1805) & Ffusc & psammophil, pholeophil & 2 \\
\hline Cetonia aurata (Linnaeus, 1758) & Cherc, Frufa & thermophil & $\begin{array}{l}3,10,11,69,81 \\
86\end{array}$ \\
\hline Liocola marmorata (Fabricius, 1792) & Lfuli & & 3,11 \\
\hline Trichius fasciatus (Linnaeus, 1758) & Mrugi & & $3,10,11$ \\
\hline Potosia cuprea* (Fabricius, 1775) & Frufa, Fprat, Fural & myrmecophil & $\begin{array}{l}2,3,69,97,111 \\
113\end{array}$ \\
\hline \multicolumn{4}{|l|}{ LYCIDAE } \\
\hline Platycis minuta (Fabricius, 1787) & Lfuli & & 1 \\
\hline Platycis cosnardi (Chevrolat, 1829) & Lfuli & & 113 \\
\hline \multicolumn{4}{|l|}{ CANTHARIDAE } \\
\hline Cantharis livida Linnaeus, 1758 & Formicidae & xerophil & 1 \\
\hline \multicolumn{4}{|l|}{ ELATERIDAE } \\
\hline Ampedus rufipennis (Stephens, 1830) & Lnige & & $1,17,113$ \\
\hline Ampedus cinnabarinus (Eschscholtz, 1829) & Lasius spp. & & 107 \\
\hline Ampedus pomorum (Herbst, 1784) & Lnige & & 96 \\
\hline Ampedus hjorti (Rye, 1905) & Lbrun & & 2,17 \\
\hline Ampedus balteatus (Linnaeus, 1758) & Lnige & & 96,107 \\
\hline Cardiophorus asellus Erichson, 1840 & Frufa & psammophil, pholeophil & 1 \\
\hline \multicolumn{4}{|l|}{ DERMESTIDAE } \\
\hline Dermestes palmiSjöberg, 1950 & Cherc & & 8,18 \\
\hline Globicornis emarginata (Gyllenhal, 1808) & Camponotus spp. & & 15 \\
\hline \multicolumn{4}{|l|}{ LYCTIDAE } \\
\hline Lyctus linearis (Goeze, 1777) & Lbrun & & 2 \\
\hline \multicolumn{4}{|l|}{ ANOBIIDAE } \\
\hline Ptinus subpilosus Sturm, 1837 & Lbrun, Lfuli & & 69 \\
\hline \multicolumn{4}{|l|}{ NITIDULIDAE } \\
\hline Epuraea terminalis (Mannerheim, 1843) & Lasius spp. & saprophil & 2 \\
\hline Amphotis marginata* (Fabricius, 1781) & Lfuli & myrmecophil & $\begin{array}{l}1,21,44,54,81 \\
113\end{array}$ \\
\hline \multicolumn{4}{|l|}{ MONOTOMIDAE } \\
\hline Monotoma quadrifoveolata Aubé, 1837 & Formica spp. & & 2 \\
\hline Monotoma conicicollis* Aube, 1837 & $\begin{array}{l}\text { Frufa, Fpoly, } \\
\text { Faqui, Fprat, } \\
\text { Fural, Flugu }\end{array}$ & myrmecophil & $\begin{array}{l}1,2,4,5,6,21,39, \\
54,56,57,69,77, \\
81,82,111,112, \\
113\end{array}$ \\
\hline
\end{tabular}


Table 1. Continued.

\begin{tabular}{|c|c|c|c|}
\hline Ant associated beetle species & Host ant & Special requirement & Ref. \\
\hline Monotoma angusticollis* (Gyllenhal, 1827) & $\begin{array}{l}\text { Frufa, Fpoly, } \\
\text { Fprat, Flugu, } \\
\text { Faqui }\end{array}$ & myrmecophil & $\begin{array}{l}1,2,5,6,21,54 \\
55,56,57,63,77 \\
81,82,111,112,113\end{array}$ \\
\hline Monotoma picipes Herbst, 1793 & Formicidae & & 2 \\
\hline Monotoma longicollis (Gyllenhal, 1827) & Formicidae & & 2 \\
\hline \multicolumn{4}{|l|}{ CRYPTOPHAGIDAE } \\
\hline Hypocoprus lathridioides Motschulsky, 1839 & Fexse & xerophil & $2,24,27$ \\
\hline Micrambe abietis (Paykull, 1798) & Frufa & mycetophil & 81 \\
\hline Cryptophagus acutangulus Gyllenhal, 1827 & Formicidae & mycetophil & 1 \\
\hline Cryptophagus fallax Balfour-Browne, 1953 & Formicidae & mycetophil & 1,113 \\
\hline Cryptophagus badius Sturm, 1845 & Lfuli & mycetophil & $1,82,113$ \\
\hline Cryptophagus fuscicornis Sturm, 1845 & Lfuli & mycetophil & 1 \\
\hline Cryptophagus labilis Erichson, 1846 & Mrugi, Lbrun & mycetophil & 2,113 \\
\hline Cryptophagus confusus Bruce, 1934 & Lbrun & mycetophil & 2,73 \\
\hline Cryptophagus intermedius Bruce, 1934 & Lbrun & mycetophil & 113 \\
\hline Cryptophagus distinguendus Sturm, 1845 & Frufa & mycetophil & $1,2,81$ \\
\hline Cryptophagus scutellatus Newman, 1834 & Formica spp. & mycetophil & 2,6 \\
\hline Spavius glaber* (Gyllenhal, 1808) & $\begin{array}{l}\text { Frufa, Fpoly, } \\
\text { Faqui, Fural }\end{array}$ & $\begin{array}{l}\text { myrmecophil, } \\
\text { mycetophil }\end{array}$ & $\begin{array}{l}2,4,5,6,21,63 \\
67,81,82,112,113\end{array}$ \\
\hline Caenoscelis ferruginea (Sahlberg, 1820) & Frufa & mycetophil & $1,94,113$ \\
\hline Caenoscelis sibirica Reitter, 1889 & Lnige & mycetophil & 113 \\
\hline Atomaria peltata Kraatz, 1853 & Formica spp. & mycetophil & 6 \\
\hline Atomaria nigriventris Stephens, 1830 & Lfuli & mycetophil & 1,113 \\
\hline Atomaria procerula Erichson, 1846 & Lbrun & mycetophil & 105 \\
\hline \multicolumn{4}{|l|}{ CERYLONIDAE } \\
\hline Cerylon histeroides (Fabricius, 1792) & Lbrun, Lfuli, Frufa & & $2,5,81$ \\
\hline Cerylon ferrugineum Stephens, 1830 & Formica rufa coll. & & 63 \\
\hline \multicolumn{4}{|l|}{ BOTHRIDERIDAE } \\
\hline Teredus cylindricus (Olivier, 1790) & Lbrun & & 1,2 \\
\hline Oxylaemus variolosus* (Dufour, 1843) & Lfuli & myrmecophil & 2 \\
\hline \multicolumn{4}{|l|}{ ENDOMYCHIDAE } \\
\hline Mycetaea subterranea (Fabricius, 1801) & Lfuli & & 81,113 \\
\hline Symbiotes latus Redtenbacher, 1849 & Lbrun, Lflav & mycetophil & $1,2,113$ \\
\hline Symbiotes gibberosus (Lucas, 1849) & Lbrun, Lfuli & mycetophil & $1,2,113$ \\
\hline Leiestes seminigra (Gyllenhal, 1808) & Lnige & & 1,2 \\
\hline \multicolumn{4}{|l|}{ COCCINELLIDAE } \\
\hline Platynaspis luteorubra (Goeze, 1777) & Lnige & xerophil & 7 \\
\hline Coccinella magnifica Redtenbacher, 1843 & Ffusc, Frufa & thermophil & $\begin{array}{l}1,2,54,69,75,95 \\
113\end{array}$ \\
\hline \multicolumn{4}{|l|}{ CORYLOPHIDAE } \\
\hline Orthoperus punctulatus Reitter, 1876 & Frufa & mycetophil & 5 \\
\hline \multicolumn{4}{|l|}{ LATRIDIIDAE } \\
\hline Enicmus transversus (Olivier, 1790) & Frufa & mycetophil & 5 \\
\hline Dienerella elongata (Curtis, 1830) & Frufa & mycetophil & 1,113 \\
\hline Dienerella clathrata (Mannerheim, 1844) & Frufa & mycetophil & 6 \\
\hline Dienerella ruficollis (Marsham, 1802) & Frufa & mycetophil & $1,81,113$ \\
\hline Stephotethus rugicollis (Olivier, 1790) & Frufa & mycetophil & 1,113 \\
\hline Corticaria longicollis ${ }^{\star}$ Zetterstedt, 1838) & $\begin{array}{l}\text { Lnige, Frufa, } \\
\text { Fprat, Fpoly, Faqui }\end{array}$ & myrmecophil, mycetophil & $\begin{array}{l}1,2,4,5,6,21,81 \\
95,105,113\end{array}$ \\
\hline Corticaria crenicollis Mannerheim, 1844 & Frufa & & 1,81 \\
\hline
\end{tabular}




\begin{tabular}{|c|c|c|c|}
\hline Ant associated beetle species & Host ant & Special requirement & Ref. \\
\hline $\begin{array}{l}\text { Corticaria inconspicua* Wollaston, } 1860 \\
\text { Corticarina fuscula (Gyllenhal, 1827) }\end{array}$ & $\begin{array}{l}\text { Frufa, Fprat } \\
\text { Frufa }\end{array}$ & $\begin{array}{l}\text { myrmecophil, mycetophil } \\
\text { mycetophil }\end{array}$ & $\begin{array}{l}1,2,113 \\
5\end{array}$ \\
\hline $\begin{array}{l}\text { MYCETOPHAGIDAE } \\
\text { Mycetophagus guadriguttatus Mueller, } 1821\end{array}$ & Lfuli & mycetophil & 1,113 \\
\hline $\begin{array}{l}\text { ADERIDAE } \\
\text { Aderus populneus (Creutzer, 1796) }\end{array}$ & Lfuli & & 113 \\
\hline $\begin{array}{l}\text { TENEBRIONIDAE } \\
\text { Myrmechixenus subterraneus* Chevrolat, } 1835\end{array}$ & $\begin{array}{l}\text { Lnige, Lfuli, Fexse, } \\
\text { Ffusc, Frufa, Fprat, } \\
\text { Fpoly, Faqui }\end{array}$ & myrmecophil & $\begin{array}{l}1,2,3,4,5,21,38 \\
81,82,97,112,113\end{array}$ \\
\hline $\begin{array}{l}\text { Opatrum sabulosum (Linnaeus, 1761) } \\
\text { Pentaphyllus testaceus (Hellwig, 1792) } \\
\text { Palorus depressus (Fabricius, 1790) }\end{array}$ & $\begin{array}{l}\text { Lnige, Ffusc } \\
\text { Lbrun } \\
\text { Frufa, Fpoly }\end{array}$ & mycetophil & $\begin{array}{l}69 \\
113 \\
3,5,6,113\end{array}$ \\
\hline $\begin{array}{l}\text { LAGRIDIIDAE } \\
\text { Scraptia fuscula Mueller, } 1821\end{array}$ & Lfuli, Lbrun, Fsang & & $1,20,113$ \\
\hline $\begin{array}{l}\text { CHRYSOMELIDAE } \\
\text { Clytra quadripunctata* (Linnaeus, 1758) }\end{array}$ & $\begin{array}{l}\text { Ffusc, Fexse, } \\
\text { Frufa, Fprat, } \\
\text { Fsang, Faqui, } \\
\text { Flugu }\end{array}$ & myrmecophil, xerophil & $\begin{array}{l}1,2,3,4,21,55 \\
56,69,81,111\end{array}$ \\
\hline $\begin{array}{l}\text { CURCULIONIDAE } \\
\text { Dryopthorus corticalis (Paykull, 1792) } \\
\text { Cossonus linearis (Fabricius, 1775) }\end{array}$ & $\begin{array}{l}\text { Lbrun, Lnige } \\
\text { Lfuli }\end{array}$ & hygrophil & $\begin{array}{l}1,69,113 \\
1,113\end{array}$ \\
\hline
\end{tabular}

with the host ant Formica rufa (166) and Lasius fuliginosus (156) (Table 2). The largest number of the host ant species (15) was found for a staphylinid beetle Drusilla canaliculata (Staphylinidae).

According to Koch (1989a, 1989b and 1992) the listed beetle species can be classified in 10 different groups by their ecological requirements (Table 1). In our data, 73 out of 369 beetles can be classified as myrmecophilous. Most of the other listed AAB species are hygrophilous (require moisture) and mycetophilous (require fungi) (Table 3).

There were 162 beetle species, which had been observed with ants only according to one reference (see Table 1). The largest number of references (20) was found for a staphylinid beetle Quedius brevis.

\section{Discussion}

In this study, we encountered a total of $369 \mathrm{AAB}$ species in Fennoscandia and Denmark, and 73 species of them are classified as myrmecophilous according to Szymzsakowski (1975) and Koch (1989a, 1989b and 1992). The total number of beetle species in Fennoscandia and Denmark is about 5000 (Lundberg \& Gustafsson 1995). Thus, we can conclude that more than $7 \%$ of the beetle fauna of Fennoscandia and Denmark can be found with ants and may belong to the ant-associated species. However, 162 species have only one observation (reference) in our list (see Table 1). This may indicate that the total number of beetle species, which are able to live with ants, would be even higher if more information was available. On the other hand, this may indicate that many beetle species may occur with ants accidentally.

There is evidence that suggests that ants reduce the numbers of other arthropods, e.g. spiders (Araneae) and harvestmen (Opiliones) (Cherix \& Bourne 1980, Skinner \& Whittaker 1981, Niemelä et al. 1992 and Laakso \& Setälä 2000). However, only a few detailed investigations or experiments 
have been published concerning the ants' impact on beetle species occurrence (see Laakso \& Setälä 1998, 2000). Laakso \& Setälä (2000) concluded that biomasses of large predatory arthropods were considerably smaller in the areas of high ant density than in the areas with few ants. However, the density of ants did not affect the total species richness of arthropods. In our opinion this is not surprising because many arthropod species in the study of Laakso \& Setälä's (2000) study seems to be ant- associated. We propose that in these kinds of studies arthropod species should be categorized to antassociated and other species, and these two groups should be dealt with separately. After this categorization it would be possible to find out whether ants affect other arthropods. Furthermore, Laakso \& Setälä (1998) noticed that, based on surface area, ant mounds harboured an order of magnitude more abundant insect fauna than the surrounding soil, the typical Coleoptera taxa in the

Table 2. List of the host ant species with the abbreviations and the numbers of the ant-associated beetle species observed with them.

\begin{tabular}{|c|c|c|}
\hline Host ant species & $\begin{array}{l}\text { Abbreviations } \\
\text { used in Table } 1\end{array}$ & $\begin{array}{c}\text { Number of ant-associated } \\
\text { beetle species }\end{array}$ \\
\hline Tapinoma erraticum Latreille, 1798 & Terra & 4 \\
\hline Myrmica rubra (Linnaeus, 1758) & Mrubr & 12 \\
\hline M. ruginodis Nylander, 1846 & Mrugi & 12 \\
\hline M. rugulosa Nylander, 1849 & Mrugu & 2 \\
\hline M. sabuleti Meinert, 1861 & Msabu & 2 \\
\hline M. scabrinodis Nylander, 1846 & Mscab & 11 \\
\hline M. sulcinodis Nylander, 1846 & Msulc & 3 \\
\hline Stenamma westwoodii, Westwood, 1840 & Swest & 1 \\
\hline Leptothorax acervorum, (Fabricius, 1793) & Lacer & 2 \\
\hline Tetramorium caespitum (Linnaeus, 1758) & Tcaes & 8 \\
\hline Camponotus vagus (Scopoli, 1763) & Cvagu & 1 \\
\hline C. herculeanus (Linnaeus, 1758) & Cherc & 4 \\
\hline C. ligniperda (Latreille, 1802) & Clign & 2 \\
\hline Lasius flavus (Fabricius, 1781) & Lflav & 14 \\
\hline L. alienus (Förster, 1850) & Lalie & 4 \\
\hline L. brunneus (Latreille, 1798) & Lbrun & 77 \\
\hline L. niger (Linnaeus, 1758) & Lnige & 42 \\
\hline L. fuliginosus (Latreille, 1798) & Lfuli & 156 \\
\hline L. umbratus (Nylander, 1846) & Lumbr & 7 \\
\hline L. mixtus (Nylander, 1846) & Lmixt & 2 \\
\hline Formica fusca (Linnaeus, 1758) & Ffusc & 23 \\
\hline F. gagatoides Ruzsky, 1904 & Fgaga & 1 \\
\hline F. lemaniBondroit, 1917 & Flema & 2 \\
\hline F. cinerea Mayr, 1853 & Fcine & 2 \\
\hline F. cunicularia, Latreille, 1798 & Fcuni & 1 \\
\hline F. rufibarbis Fabricius, 1793 & Frufi & 5 \\
\hline F. exsecta Nylander, 1846 & Fexse & 31 \\
\hline F. suecica Adlerz, 1902 & Fsuec & 3 \\
\hline F. uralensis Ruzsky, 1895 & Fural & 11 \\
\hline F. sanguinea Latreille, 1798 & Fsang & 20 \\
\hline F. truncorum Fabricius, 1804 & Ftrun & 7 \\
\hline F. rufa Linnaeus, 1761 & Frufa & 166 \\
\hline F. polyctena Förster, 1850 & Fpoly & 25 \\
\hline F. aquilonia Yarrow, 1955 & Faqui & 29 \\
\hline F. lugubris Zetterstedt, 1840 & Flugu & 12 \\
\hline F. pratensis Retzius, 1783 & Fprat & 34 \\
\hline F. nigricans Emery, 1909 & Fnige & 4 \\
\hline Polyergus rufescens (Latreille, 1798) & Prufe & 1 \\
\hline
\end{tabular}


mounds being Ptilidae and Staphylinidae. As many of the listed $\mathrm{AAB}$ species belong to these two families, our study provides some support for this finding (see Table 1).

Koch (1989a, 1989b and 1992) has categorized beetle species according to their ecological requirements. Based on this classification, we divided ant-associated beetles into two groups: myrmecophilous species and other ant-associated species. Other ant-associated species are regularly found with ants, but based on Koch (1989a, 1989b, 1992) they are not necessarily dependent on ants. Koch (1989a, 1989b, 1992) has also categorized some beetle species as myrmeco- or mycetophagous (feed upon ants or fungus). We have categorized these species as myrmeco- or mycetophilous species, since they are clearly dependent on ants or fungus as a food resource. Also one mycetobiont (bounded to fungus) species is classified as mycetophilous. Furthermore, according to Szymzsakowski (1975) the endemic Nordic beetle species Eocatops lapponicus is classified as myrmecophilous too.

Vaz-De-Mello et al. (1998) have studied rare or poorly known beetle species of the family Scaradaeidae and propose that myrmecophilous interactions between beetles and ants are possibly more common than has been thought previously. We agree with Vaz-De-Mello et al. (1998) and state that many beetles, which are not previously known to benefit from ants, do so, however. According to present knowledge, it is difficult to specify which listed $\mathrm{AAB}$ species could be myrmecophiles.
A generally accepted classification of myrmecophilous arthropods is based on a series of works by Wasmann (e.g. Wasmann 1910, translated into English by Wheeler in 1910). Wasmann devised 5 behavioural categories: (1) synechthrans (persecuted guests), (2) synoeketes (indifferently tolerated guests), (3) symphiles (true guests), (4) ectoparasites and endoparasites and (5) trophobionts (provide secretions to the ants). In the current study, we list beetle species that are classified as myrmecophilous according to Szymczakowski (1975) and Koch (1989a, 1989b, 1992). As the ecology of these species is poorly known, we did not classify the species into the appropriate behavioural categories. Correspondingly, Larsson (1943) has classified ant-associated species by their behaviour to three categories; synechthrans, synoeketes and symphiles. All the beetle species belonging to these behavioural categories are myrmecophilous according to Hölldobler and Wilson's (1990) definition. Thus, there are many myrmecophilous beetles in Larsson's (1943) list that are not classified myrmecophilous according to Koch (1989a, 1989b, 1992). It seems that at least both Larsson's (1943) and Johansen's (1904) description of myrmecophilous beetles is probably different from Koch's (1989a, 1989b, 1992) and Hölldobler \& Wilson's (1990). We propose that when a beetle species is found to associate with ants but knowledge of its basic ecology is lacking, the term ant-associated beetle (AAB) species should be used instead of the term myrmecophilous.

Table 3. The number of $A A B$ species found with each host ant genus. AAB species are classified according to their ecological requirements. The same AAB species can have zero, one or two special requirements.

AAB species' special requirement

\begin{tabular}{|c|c|c|c|c|c|}
\hline Host ant genus & Hygrophil & Mycetophil & Myrmecophil & Xerophil & Other groups \\
\hline Tapinoma spp. & 0 & 0 & 1 & 1 & 0 \\
\hline Myrmica spp. & 9 & 2 & 10 & 8 & 0 \\
\hline Stenamma spp. & 0 & 0 & 1 & 1 & 0 \\
\hline Leptothorax spp. & 0 & 0 & 2 & 2 & 0 \\
\hline Tetramorium spp. & 1 & 0 & 4 & 5 & 1 \\
\hline Camponotus spp. & 1 & 1 & 5 & 0 & 1 \\
\hline Lasius spp. & 45 & 25 & 54 & 12 & 15 \\
\hline Formica spp. & 37 & 36 & 56 & 15 & 14 \\
\hline Polyergus spp. & 0 & 0 & 0 & 1 & 0 \\
\hline Total & 93 & 64 & 133 & 45 & 31 \\
\hline
\end{tabular}


Both $\mathrm{AAB}$ and most of the host-ant species are often difficult to identify. The largest number of listed AAB species exists with Formica rufa. Probably, at least in some older studies, most of the so-called $F$. rufa-group species ( $F$. rufa, $F$. polyctena, $F$. aquilonia, $F$. lugubris and $F$. pratensis) have been incorrectly identified as $F$. rufa. Moreover the species $F$. aquilonia was not described until 1955 by Yarrow. This may partly explain why six times more AAB-species have been observed with $F$. rufa than with $F$. aquilonia, although the latter probably is the most common mound-building wood ant in Fennoscandia. Indeed, Päivinen (1999) found in Formica aquilonia mounds 20 ant-associated beetle species not previously recorded for $F$. aquilonia. In total, only $10 \%$ of AAB-species that Päivinen (1999) found in $F$. aquilonia's mounds were earlier observed with this species.

64 ant species have been recorded in Fennoscandia and Denmark (Collingwood 1979). According to our study, AAB species were found with only $2 / 3$ of them. Due to the poor knowledge of AAB species living with ants (see Päivinen 1999), we assume that clearly more AAB species could further be found with most of the ant species. To find more AAB species in the future, research should be focused on those ant species that do not exist on the present list.

Finally, we conclude that ant colonies are species rich habitats for many beetles in Fennoscandia and Denmark. In addition, myrmecophilous interactions between beetles and ants can be more frequent than previously thought. Despite the fact that ants have been shown to have negative association with many arthropods, ants seem to have an important role in maintaining beetle species diversity. We recommend the use of the term antassociated beetle (AAB) for beetles, which are found to live with ants. More detailed studies on the basic ecology of ant-beetle interactions should be done to determine which species are true myrmecophiles.

Acknowledgements. Special thanks to Veli-Matti Mukkala and Ilpo Rutanen, who gave us their valuable field observations concerning about ants and beetles. We are also grateful to Teija Virola, Esko Hyvärinen, Jouni Laakso, Heikki Setälä, Jouni Sorvari, Lotta Sundström and Tero Toivanen for improving the manuscript. Special thanks also to Tom Clayhills, Jyrki Muona and Juha Siitonen who helped us to solve many taxonomical problems and Pekka Punttila for helping us in the literature survey. The study was financed by the Jenny and Antti Wihuri Foundation, University of Jyväskylä, Otto A. Malm Foundation, the Societas pro Fauna et Flora Fennica and the Entomological Society of Finland.

\section{References}

Adlerz, G. 1911: Cetonia aurata och Trichius fasciatus i myrbon. - Ent. Tidskr. 32: 43-46.

Adlerz, G. 1912: Resa till Öland sommaren. — Ent. Tidskr. 33: 43-46.

Adlerz, G. 1913: Myrornas liv. — Ljus, Stockholm. 243 p.

Andersen, J., Nielsen, T. R. \& Zachariassen, K. E. 1984: Nye funn av biller i Norge. - Fauna Norv. Ser. B. 31: 59-60.

Andersson, B. 1977: Notiser om svenska skalbaggar. Ent. Tidskr. 98: 97-102.

Andersson, B. 1981: Notiser om svenska skalbaggar 3. Ent. Tidskr. 102: 141-146.

Aurivillius, C. 1917: Skalbaggar I. Coleoptera. Växtbaggar. Phytophaga. - Svensk Insektfauna 9. Almqvist \& Wiksells Boktryckeri-A-B, Uppsala. 119 pp.

Aurivillius, C. 1920: Skalbaggar II. Coleoptera. Snytbaggar. Rhynchopora. - Svensk Insektfauna 9. Almqvist \& Wiksells Boktryckeri-A-B, Uppsala. 64 pp.

Bangsholt, F. 1981: Femte tillaeg til "Fortegnelse over Danmarks biller" (Coleoptera). - Ent. Medd. 48: 49-103.

Baranowski, R. 1975: Några bidrag till kännedomen om coleopterfaunan vid nedre Dalälven. - Ent. Tidskr. 96: 97-115.

Baranowski, R. 1976: Några för Sverige nya skalbaggar (Coleoptera). — Ent. Tidskr. 97: 117-123.

Baranowski, R. 1979: Intressanta skalbaggsfynd 4. — Ent. Tidskr. 100: 71-80.

Baranowski, R. 1980a: Några bidrag till kännedomen om coleopterfaunan vid nedre Dalälven. 2. — Ent. Tidskr. 101: 29-42.

Baranowski, R. 1980b: Intressanta skalbaggsfynd 5. — Ent. Tidskr. 101: 99-106.

Baranowski, R. 1982: Intressanta skalbaggsfynd 6. — Ent. Tidskr. 103: 130-136.

Cherix, D. \& Bourne, J. D. 1980: A field study on a supercolony of the Red wood ant Formica lugubris Zett. in relation to other predatory arthropods (spiders, harvestman and ants). - Rev. Suisse. Zool. 87: 955-973.

Clayhills, T. 1988: Tiedonantoja; Oxypoda lapponica tavattu Suomesta (Staphylinidae). — Not. Entomol. 68: 151.

Collingwood, C. A. 1957: Myrmecophilous beetles in the Midlands. - Entomol. Rec. J. Var. 69: 9-14.

Collingwood, C. A. 1959: Notes on Irish Coleoptera. Ent. Gaz. 10: 39-42.

Collingwood, C. A. 1965: Myrmecophilous Beetles in Ireland, Scotland and Wales. - Entomol. Rec. J. Var. 77: 45-47. 
Collingwood, C. A. 1979: The Formicidae (Hymenoptera) of Fennoscandia and Denmark. — Fauna Ent. Scand. 8: $1-175$.

Donisthorpe, H. 1927: The guests of British ants, their habits and life-histories. - George Routledge and Sons, London. 244 pp.

Douglas, J. W. 1858: Ants' nest beetles. - Zoologist 16: 6067-6068.

Ehnström, B. 1983: Faunistiska anteckningar om trädskalbaggar. — Ent. Tidskr. 104: 76-79.

Franc, V. 1992: Myrmecophilous beetles of Slovakia with special reference to their endangerment and perspectives for protection. - Acta Universitatis Carolinae Biologica 36: 299-324.

Gillerfors, G. 1982: Anteckningar om svenska coleoptera 2. - Ent. Tidskr. 103: 73-77.

Gillerfors, G. 1990: Anteckningar om svenska coleoptera 3. - Ent. Tidskr. 111: 87-89.

Hansen, M. 1988: Syvende tillage til "Fortegnelse over Danmarks biller" (Coleoptera). — Ent. Medd. 56: $131-155$.

Hansen, M. \& Mahler, V. 1985: Nogle billearter, nye for den danske fauna (Coleoptera). — Ent. Medd. 53: 1-23.

Hansen, M., Mahler, V., Palm, E. \& Vagtholm-Jensen, O. 1990: Ottende tillaeg til "Fortegnelse over Danmarks Biller" (Coleoptera). — Ent. Medd. 58: 11-29.

Hansen, M., Mahler, V., Palm, E. \& Vagtholm-Jensen, O. 1991: Niende tillaeg til "Fortegnelse over Danmarks Biller" (Coleoptera). — Ent. Medd. 59: 5-21.

Hansen, M., Mahler, V., Pritzl, G. \& Runge, J. B. 1994: 13. tillaeg til "Fortegnelse over Danmarks Biller" (Coleoptera). — Ent. Medd. 62: 65-89.

Hansen, V. 1950: Biller XIII. Clavicornia 1. — Danmarks Fauna 55. G. E. C. Gads Forlag, Kobenhavn. 278 pp.

Hansen, V. 1951a: Biller VIV. Clavicornia 2. — Danmarks Fauna 56. G. E. C. Gads Forlag, Kobenhavn. 253 pp.

Hansen, V. 1951b: Biller XV. Rovbiller 1. — Danmarks Fauna 57. G. E. C. Gads Forlag, Kobenhavn. 274 pp.

Hansen, V. 1952: Biller XVI. Rovbiller 2. — Danmarks Fauna 58. G. E. C. Gads Forlag, Kobenhavn. 251 pp.

Hansen, V. 1954: Biller XVII. Rovbiller 3. - Danmarks Fauna 59. G. E. C. Gads Forlag, Kobenhavn. 499 pp.

Hansen, V. 1956: Biller XVIII. Barkbiller. - Danmarks Fauna 62. G. E. C. Gads Forlag, Kobenhavn. 196 pp.

Hansen, V. 1957: Biller XIX. Almindelig del. — Danmarks Fauna 63. G. E. C. Gads Forlag, Kobenhavn. 248 pp.

Hansen, V. 1958: Biller XX. Tilleagsbind. - Danmarks Fauna 64. G. E. C. Gads Forlag, Kobenhavn. 244 pp.

Hansen, V. 1964: Fortegnelse over Danmarks biller (Coleoptera). — Ent. Medd. 33: 1-507.

Hansen, V. 1965: Biller XXI. Snudebiller. - Danmarks Fauna 69. G. E. C. Gads Forlag, Kobenhavn. 524 pp.

Hansen, V. 1966a: Biller XXII. Traebukke. — Danmarks Fauna 73. G. E. C. Gads Forlag, Kobenhavn. 228 pp.

Hansen, V. 1966b: Biller XXIII. Smaeldere og Praktbiller. - Danmarks Fauna 74. G. E. C. Gads Forlag, Kobenhavn. $179 \mathrm{pp}$.

Hansen, V. 1967: Nye danske biller (Coleoptera) 1966.
Ent. Medd. 35: 218-222.

Hansen, V. 1968a: Biller XXIV. Sandspringere og Lobebiller. - Danmarks Fauna 76. G. E. C. Gads Forlag, Kobenhavn. 451 pp.

Hansen, V. 1968b: Biller XXV. Ådselbiller og Stunpbiller. — Danmarks Fauna 77. G. E. C. Gads Forlag, Kobenhavn. 353 pp.

Hansen, V. 1968c: Nye danske biller (Coleoptera) 1967. Ent. Medd. 36: 409-413.

Hansen, V. 1969: Biller XXVI. Andet tillaegsbind. Danmarks Fauna 78. G. E. C. Gads Forlag - Kobenhavn. 128 pp.

Hansen, V. 1970: Nye danske biller (Coleoptera) 1969. Ent. Medd. 38: 165-169.

Hansen, V. 1971: Billefaunan i Jaegersborg Dyrehave (Coleoptera). — Ent. Medd. 39: 161-200.

Hansen, V. 1973a: Biller VIII. Vandkalve. — Danmarks Fauna 34. G. E. C. Gads Forlag, Kobenhavn. 248 pp.

Hansen, V. 1973b: Biller IX. Vandkaerer. — Danmarks Fauna 36. G. E. C. Gads Forlag, Kobenhavn. 172 pp.

Hansen, V. 1973c: Biller X. Blödvinger. — Danmarks Fauna 44. G. E. C. Gads Forlag, Kobenhavn. 344 pp.

Hansen, V. 1973d: Biller XII. Heteromerer. — Danmarks Fauna 50. G. E. C. Gads Forlag, Kobenhavn. 307 pp.

Hansen, V. \& Henriksen, K. 1927: Biller VII. Bladbiller og Bonnebiller. - Danmarks Fauna 31. G. E. C. Gads Forlag, Kobenhavn. 401 pp

Holstebroe, H. O. 1910: De danske Arter af Slaegten Choleva Latreille. — Ent. Medd. 2(3-4): 377-403.

Huggert, L. 1967: Några sällsyntare Coleoptera. — Ent. Tidskr. 88: 170-173.

Huggert, L. \& Ulefors, S.-O. 1971: Anteckningar om svenska Coleoptera. — Ent. Tidskr. 92: 54-65.

Hölldobler, B. \& Wilson, E. O. 1990: The Ants. — SpringerVerlag, Berlin, Heidelberg. 732 pp.

Johansen, J. P. 1895-96: Catalogus Coleopterorum Danicorum Fam. Staphylinidae. Pars 4. — Ent. Medd. 3: 235-276.

Johansen, J. P. 1903: Meddelelse af Fund av adskillige for Faunaen nye og af nogle kjendte, sjaeldne Biller. Ent. Medd. 2(3-4): 180-138.

Johansen, J. P. 1904: Om Undersogelse af Myretuer samt fortegnelse over de i Danmark fundne, saakaldte myrmecophile Biller. — Ent. Medd. 2: 217-265.

Johansen, J. P. 1906: Meddelelse om Fund av adskillige for Faunaen nye og af nogle kjendte, sjaeldne Billeder. 3 . — Ent. Medd. 2(3-4): 65-84.

Johnson, C. 1988: Notes on some British Cryptophagus Herbst (Coleoptera: Cryptophagidae), including confusus bruce new to Britain. - Ent. Gaz. 329-335.

Jorum, P. 2000: Billefaunaen i Hald Egeskov (Coleoptera). — Ent. Medd. 68: 1-46.

Kangas, E. 1938: Revision der finnischen Arten der Gattung Oligota Mann. (Col., Staphylinidae). - Ann. Ent. Fenn. 4: 201-212.

Kangas, E. 1951: Die finnischen Euplectus-Arten (Col., Pselaphidae). - Ann. Ent. Fenn. 17: 136-148.

Kangas, E. 1982: Uber einige Arten der Oligota pusillima 
(Gravenhorst) - Gruppe (Coleoptera, Staphylinidae). — Ann. Ent. Fenn. 48: 65-70.

Kangas, E. 1983: Eine neue Oxypoda-Art (Coleoptera, Staphylinidae) aus Finnland. - Ann. Ent. Fenn. 49: 54-56.

Kistner, D. H. 1982: The social insects' bestiary. - In: Hermann, H. R. (ed.), Social Insects. Academic Press, New York. 1-244 pp.

Kistner, D. H., Weissflog, A., Rosciszewski, K \& Maschwitz, U. 1997: New species, new genera, and new records of myrmecophiles associated with army ants (Aenictus sp.) with the description of a new subtribe of Staphylinidae (Coleoptera; Formicidae: Aenictinae). Sociobiology 29: 121-221.

Koch, K. 1989a: Ökologie 1. — Die Käfer Mitteleuropas E1. $440 \mathrm{pp}$.

Koch, K. 1989b: Ökologie 2. — Die Käfer Mitteleuropas E2. $382 \mathrm{pp}$.

Koch, K. 1992: Ökologie 3. - Die Käfer Mitteleuropas E3. 389 pp.

Kornerup, U. 1960: 5. Coleoptera — Biller. — Ent. Medd. 30: 59-104.

Krogerus, R. 1934: Tiedonantoja yhdistyksistä; Thiasophila bercionis. — Not. Entomol. 14: 115.

Kryger, J. P. \& Sonderup, H. P. S. 1945: Biologiske Iagttagelser over 200 Arten af danske Billelarven. 2. — Ent. Medd. 24: 175-261.

Laakso, J. \& Setälä, H. 1997: Nest mounds of red wood ant (Formica aquilonia): hot spots for litter-dwelling earthworms. - Oecologia 111: 565-569.

Laakso, J. \& Setälä, H. 1998: Composition and trophic structure of detrital food web in ant nest mounds of Formica aquilonia and in the surrounding forest soil. _ Oikos 81: 266-278.

Laakso, J. \& Setälä, H. 2000: Impacts of wood ants (Formica aquilonia Yarr.) on the invertebrate food web of the boreal forest floor. - Ann. Zool. Fennici 37: 93-100.

Landin, B.-O. 1957: Skalbaggar (Coleoptera). Bladhorningar (Lamellicornia). Fam. Scarabaeidae. Svensk Insektfauna 46. Almqvist \& Wiksells Boktryckeri-A-B, Uppsala. 155 pp.

Larsson, S. G. 1943: Myrer. — Danmarks Fauna 49. G. E. C. Gads Forlag, Kobenhavn. 190 pp.

Lindberg, H. 1943: Nykomlingar till Finlands skalbaggsfauna. - Not. Entomol. 23: 50-58.

Lindgren, L. A. H. 1945: Entomologiska notiser 2. - Ent. Tidskr. 66: 73-78.

Lindroth, C. H. 1933: Skalbaggar (Coleoptera). Oligfotade baggar. Heteromera. - Svensk Insektfauna 27. Almqvist \& Wiksells Boktryckeri AB, Uppsala. 158 pp.

Lindroth, C. H. 1946: Våra skalbaggar och hur man känner igen dem. Del 1. Markens och vattnets skalbaggar jordlöpare, vattenbaggar och kortvingar. —Albert Bonniers Förlag, Stockholm. 81 pp.

Lindroth, C. H. 1961: Skalbaggar (Coleoptera). Sandjägare och Jordjöpare. Fam. Carabidae. - Svensk Insektfauna 46. Almqvist \& Wiksells Boktryckeri AB, Uppsala. 155 pp.
Lovedal, E. A. 1891-92: Fortegnelse over de I Danmark levande Cryptophagidae og Lathridiidae. — Ent. Medd. 3: 235-276.

Lundberg, S. 1961: Bidrag till kännedom om svenska Coleoptera. 4. - Ent. Tidskr. 82: 64-68.

Lundberg, S. 1972: Bidrag till kännedom om svenska skalbaggar. 13. - Ent. Tidskr. 93: 42-56.

Lundberg, S. 1973: Bidrag till kännedom om svenska skalbaggar. 14. - Ent. Tidskr. 94: 28-33.

Lundberg, S. 1976: Bidrag till kännedom om svenska skalbaggar. 16 (Coleoptera). — Ent. Tidskr. 97: 15-20.

Lundberg, S. 1977: Fynd av två Norden nya skalbaggsarter (Coleoptera). - Ent. Tidskr. 98: 5-6.

Lundberg, S. 1978a: Bidrag till kännedom om svenska skalbaggar. 17 (Coleoptera). — Ent. Tidskr. 99: 31-34.

Lundberg, S. 1978b: Skalbaggsarter, som inte återfunnits i Sverige på lång tid — några tips (Coleoptera). — Ent. Tidskr. 99: 121-126.

Lundberg, S. 1980: Oxypoda scanica n.sp. and O. lapponica n. sp. from Sweden (Coleoptera: Staphylinidae). - Ent. Scand. 11: 348-352.

Lundberg, S. 1981: Sällsynta skalbaggar i Halltorps hage. — Ent. Tidskr. 102: 134-135.

Lundberg, S. 1983: Skalbaggar på Ölands Stora alvar. — Ent. Tidskr. 104: 121-126.

Lundberg, S. 1984: Bidrag till kännedom om svenska skalbaggar 22. — Ent. Tidskr. 105: 107-108.

Lundberg, S. 1993: Sällsynta och hotade skalbaggar i Hornsö- och Strömsrumstrakten i östra Småland. Ent. Tidskr. 114: 83-96.

Lundberg, S. \& Gustafsson, B. 1995: Catalogus Coleopterorum Sueciae. - Naturhistoriska riksmuseet \& Entomologiska föreningen i Stockholm.

Mahler, V. 1987: Sjette tillaed til "Fortegnelse over Danmarks biller" (Coleoptera). — Ent. Medd. 54: 181-235.

Martin, O. 1989: Smaeldere (Coleoptera, Elateridae) fra gammel lovskov i Danmark. — Ent. Medd. 57:1-110.

Meinert, F. 1887-88a: Scydmaenus - Larven. — Ent. Medd. 1: 144-150.

Meinert, F. 1887-88b: Catalogus Coleopterorum Danicorum Fam. Staphylinidae. Pars 1. — Ent. Medd. 1: 215-284.

Meinert, F. 1889-90: Catalogus Coleopterorum Danicorum Fam. Staphylinidae. Pars 2. — Ent. Medd. 2: 227-279.

Niemelä, J., Haila, Y., Halme, E., Pajunen, T. \& Punttila, P. 1992: Small-scale heterogeneity in the spatial distribution of Carabid beetles in the Southern Finnish taiga. — J. Biogeogr. 19: 173-181.

Nilssen, A. C. and Andersen, J. 1977: Funn av Coleoptera fra Nord-Norge. — Norw. J. Entomol. 24: 7-9.

Owen, J. A. 1986: Formica aquilonia Yarrow (Hym. Formicidae) and some beetle associates in the Isle of Skye. — Entomol. Mon. Mag. 122: 120.

Owen, J. A. 2000: Coleoptera occurring underground at the roots of old trees. - Ent. Gaz. 51: 239-256.

Päivinen, J. 1999: Effects of selective logging on beetles living in ant nests. - M.Sc. thesis, University of Jyväskylä, Jyväskylä. 24 pp. [In Finnish with English abstract]. 
Palm. T. 1936: Coleoptera i bivråk- och ormvråkbon. Ent. Tidskr. 57: 84-96.

Palm, T. 1943: Anteckningar om svenska skalbaggar. — Ent. Tidskr. 64: 74-85.

Palm, T. 1946: Coleopterfaunan i jämtländsk lavgranskog. — Ent. Tidskr. 67: 109-139.

Palm, T. 1947: Anteckningar om svenska skalbaggar. III. — Ent. Tidskr. 68: 171-178.

Palm, T. 1948: Skalbaggar (Coleoptera). Kortvingar: Fam. Staphylinidae. Underfam. (Micropeplinae, Phloeocharinae, Olisthaerinae, Proteininae, Omaliinae). Svensk Insektfauna 38. Almqvist \& Wiksells Boktryckeri AB, Uppsala. 209 pp.

Palm, T. 1954a: Anteckningar om svenska skalbaggar. IX. — Ent. Tidskr. 75: 13-28.

Palm, T. 1954b: Bidrag till kännedomen om svenska skalbaggars biologi och systematik. — Ent. Tidskr. 75: 151-161.

Palm, T. 1956: Anteckningar om svenska skalbaggar. XI. — Ent. Tidskr. 77: 56-63.

Palm, T. 1959: Bidrag till kännedomen om svenska skalbaggars biologi och systematik. 28-35. - Ent. Tidskr. 80: 22-32.

Palm, T. 1961: Skalbaggar. Coleoptera. Kortvingar: Fam. Staphylinidae. Underfam. Oxytelinae, Oxyporinae, Steninae, Euaesthetinae. Häfte 2. - Svensk Insektfauna 48. Almqvist \& Wiksells Boktryckeri AB, Uppsala. 126 pp.

Palm, T. 1963: Skalbaggar. Coleoptera. Kortvingar: Fam. Staphylinidae. Underfam. Paederinae, Staphylininae. Häfte 3. - Svensk Insektfauna 49. Almqvist \& Wiksells Boktryckeri AB, Uppsala. 168 pp.

Palm, T. 1966: Skalbaggar. Coleoptera. Kortvingar: Fam. Staphylinidae. Underfam. Habrocerinae, Trichophyinae, Tachyporinae. Häfte 4. - Svensk Insektfauna 50. Almqvist \& Wiksells Boktryckeri AB, Uppsala. 93 pp.

Palm, T. 1968: Skalbaggar. Coleoptera. Kortvingar: Fam. Staphylinidae. Underfam. Aleocharinae (DeinopsisTrichomicra). Häfte 5. - Svensk Insektfauna 51. Almqvist \& Wiksells Boktryckeri AB, Uppsala. 112 pp.

Palm, T. 1970: Skalbaggar. Coleoptera. Kortvingar: Fam. Staphylinidae. Underfam. Aleocharinae (Atheta). Svensk Insektfauna 52. Almqvist \& Wiksells Boktryckeri AB, Uppsala. 215 pp.

Palm, T. 1972: Skalbaggar. Coleoptera. Kortvingar: Fam. Staphylinidae. Underfam. Aleocharinae (AleuonotaTinotus). - Svensk Insektfauna 55. Almqvist \& Wiksells Boktryckeri AB, Uppsala. 148 pp.

Palm, T. 1979: Om skalbaggsfaunan i komposthögar vid Uppsala. — Ent. Tidskr. 100: 33-36.

Palm, T. 1985a: Skalbaggar i en gammal tallskog i Uppsala. — Ent. Tidskr. 106: 107-112.

Palm, T. 1985b: Skalbaggsstudier på en uppländsk mosse. — Ent. Tidskr. 106: 138-143.

Palmen, E. 1936: Pikkutietoja; eräitä mielenkiintoisia kovakuoriaislöytöjä. — Ann. Ent. Fenn. 2: 149-151. [In Finnish].

Paulsen, O. 1991: Euthiconus conicicollis (Fairm. \&
Laboulbene, 1855) (Col., Scydmaenidae) og Anitys rubens (Hoffmann, 1803) (Col., Anobiidae) nye arter i Norge. - Fauna Norv. Ser. B. 38: 31.

Persson, B. 1981: Nya landskapsfynd av skalbaggar. — Ent. Tidskr. 102: 43-45.

Pritzl, G. \& Mahler, J. 1982: De danske Oligota — arter (Coleoptera: Staphylinidae). —Ent. Medd. 49: 107-112.

Rosenberg, E. C. 1913: Bidrag til Kundskapen om Billernes Levevis, Udvikling og Systematic. 3. Undersogelser over Danmarks Billefauna I Dyreboer, saerlig underjordiske. — Ent. Medd. 2(5): 37-76.

Rosenberg, E. C. 1914: Mindre Meddelelser. Nye og sjaeldne danske Biller. — Ent. Medd. 2(5): 118-123.

Rosenberg, E. C. 1024: Contributions to the knowledge of the life-habits, development and systematics of the Coleoptera. 4. On the larva Batrisodes venustus Reichenb., with remarks on the life-habits of other socalled myrmecophile Coleoptera. - Ent. Medd. 14: 374-388.

Rosengren, R., Fortelius, W., Lindström, K. and Luther, A. 1987: Phenology and causation of nest heating and thermoregulation in red wood ants of the Formica rufa group studied in coniferous forest habitats in southern Finland. - Ann. Zool. Fenn. 24: 147-155.

Rosengren, R \& Sundström, L. 1991: The interaction between red wood ants, Cinara aphids, and pines. A ghost of mutualism past? - In: Huxley, C. R. \& Cutler, D. F. (eds.), Ant-Plant Interactions. Oxford University Press, Oxford. 80-91 pp.

Rydh, I. 1977: Nyfynd av skalbaggar i Blekinge och Småland 2 (Coleoptera). — Ent. Tidskr. 98: 141-142.

Sagvolden, B. A. and Hansen, L. O. 1996: Notes on Norwegian Coleoptera. 3. - Fauna Norv. Ser. B. 43: 89-94.

Schlick, W. 1895-96: Biologiske Bidrag. — Ent. Medd. 5: 110-138.

Schlick, W. 1897: Biologiske Bidrag. Coleoptera 3. Fortsaettelse. — Ent. Medd. 2(1): 49-67.

Siitonen, J. 1993: Faunistic records of Carabidae and Staphylinidae (Coleoptera) caught by pitfall trapping in western Finnish Lapland. - Ent. Fenn. 4: 225-231.

Skidmore, P. and Johnson, C. 1969: A preliminary list of the Coleoptera of Merioneth, North Wales. - Ent. Gaz. 20: $139-225$.

Skinner, G. J. and Whittaker, J. B. 1981: An experimental investigation of interrelationship between the wood-ant (Formica rufa) and some tree-canopy herbivores. - J. Anim. Ecol. 50: 313-326.

Sloggett, J. J., Manica, A., Day, M. J. and Majerus, M. E. N. 1999: Predation of ladybirds (Coleoptera: Coccinellidae) by wood ants, Formica rufa L. (Hymenoptera: Formicidae). — Ent. Gaz. 50: 217-221.

Spessivtseff, P. 1925: Andra familjegruppen snytbaggar (Rhynchophora). Skalbaggar (Coleoptera). Barkborrar (Scolytidae). - Svensk Insektfauna 28: 194 pp. Almqvist \& Wiksells Boktryckeri-A-B, Uppsala.

Szymczakowski, Von W. 1975: Unerwarteter Fund einer neuen Eocatops-Art in Schweden und Finnland (Col. Catopidae). — Ent. Tidskr. 96: 3-7. 
Sörensson, M. 1979: Uppgifter om svenska skalbaggar. Ent. Tidskr. 100: 67-69.

Sörensson, M. 1996: Sydsvenska kortvingar (Coleoptera. Staphylinidae) ur ett naturvårdsperspektiv: 1. Quedius truncicola. — Ent. Tidskr. 117: 11-22.

Vallenduuk, H. J. 1987: Faunistics and biology of myrmecophilous Histeridae in the Netherlands (Coleoptera). Greater than or equal to. - Entomol. Ber. (Amst.) 47: 53-59.

Vaz-De-Mello, F. Z., Louzada, J. N. C \& Schoereder, J. H. 1998: New Data and Comments on Scarabaeidae (Coleoptera: Scarabaeoidea) Associated with Attini (Hymenoptera: Formicidae). — Coleopt. Bull. 52: 209-216.

Völkl, W. 1995: Behavioural and morphological adaptations of the coccinellid, Platynaspis luteorubra for exploiting ant-attended resources (Coleoptera: Coccinellidae). - J. Insect Behav. 8: 653-670.

Wasmann, E. 1910: Die Ameisen und ihre Gäste. Memoires 2: 209-232.

Wegelius, A. 1960: Bidrag till kännedomen om skalbaggsfaunan inom Pallas-Ounastunturi nationalpark. — Not.
Entomol. 40: 86-107.

West, A. 1913: Mindre meddelelser. — Ent. Medd. 2(5): 29-32.

West, A. 1930: Tillaeg og Rettelser til Fortegnelserne over de danske Coleoptera. — Ent. Medd. 16: 441-492.

Wheeler, W. M., 1910: Ants, their structure, development and behaviour. - Columbia Univ. Press, New York. 663 pp.

Whitehead, P. F. 1996: The northernmost British record of Euryusa sinuata Erichsin, 1837 (Coleoptera: Staphylinidae). — Ent. Gaz. 47: 255-256.

Widenfalk, T. 1954: Smärre meddelanden och notiser. Fynd av några sällsyntare eller Sverige nya skalbaggar. Ent. Tidskr. 75: 61-62.

Wilson, E. O. 1971: The insect societies. — Belknapp Press of Harward University Press, Cambridge, Mass. 548 pp.

Wojcik, D. P. 1990: Behavioral interactions of fire ants and their parasites, predators and inquilines. - In: Van der Meer, R. K., Jaffe, K. \& Cedeno, A (eds.), Applied Myrmecology: A World Perspective. Westview Press, Boulder, San Francisco \& Oxford. 329-344 pp. 